\title{
The inner wind of IRC+10216 revisited: new exotic chemistry and diagnostic for dust condensation in carbon stars ${ }^{\star}$
}

\author{
I. Cherchneff
}

\author{
Departement Physik, Universität Basel, Klingelbergstrasse 82, 4056 Basel, Switzerland \\ e-mail: isabelle.cherchneff@unibas.ch
}

Received 29 November 2011 / Accepted 26 April 2012

\begin{abstract}
Aims. We model the chemistry of the inner wind of the carbon star IRC+10216 and consider the effects of periodic shocks induced by the stellar pulsation on the gas to follow the non-equilibrium chemistry in the shocked gas layers. We consider a very complete set of chemical families, including hydrocarbons and aromatics, hydrides, halogens, and phosphorous-bearing species. Our derived abundances are compared to those for the latest observational data from large surveys and the Herschel telescope.

Methods. A semi-analytical formalism based on parameterised fluid equations is used to describe the gas density, velocity, and temperature from $1 R_{\star}$ to $5 R_{\star}$. The chemistry is described using a chemical kinetic network of reactions and a set of stiff, ordinary, coupled differential equations is solved.

Results. The shocks induce an active non-equilibrium chemistry in the dust formation zone of IRC +10216 where the collision destruction of $\mathrm{CO}$ in the post-shock gas triggers the formation of O-bearing species such as $\mathrm{H}_{2} \mathrm{O}$ and $\mathrm{SiO}$. Most of the modelled molecular abundances agree very well with the latest values derived from Herschel data on IRC+10216. The hydrides form a family of abundant species that are expelled into the intermediate envelope. In particular, HF traps all the atomic fluorine in the dust formation zone. The halogens are also abundant and their chemistry is independent of the $\mathrm{C} / \mathrm{O}$ ratio of the star. Therefore, $\mathrm{HCl}$ and other $\mathrm{Cl}$-bearing species should also be present in the inner wind of O-rich AGB or supergiant stars. We identify a specific region ranging from $2.5 R_{\star}$ to $4 R_{\star}$, where polycyclic aromatic hydrocarbons form and grow. The estimated carbon dust-to-gas mass ratio derived from the mass of aromatics formed ranges from $1.2 \times 10^{-3}$ to $5.8 \times 10^{-3}$ and agrees well with existing values deduced from observations. This aromatic formation region is situated outside hot layers where $\mathrm{SiC}_{2}$ is produced as a bi-product of silicon carbide dust synthesis. The $\mathrm{MgS}$ grains can form from the gas phase but in lower quantities than those necessary to reproduce the strength of the $30 \mu \mathrm{m}$ emission band. Finally, we predict that some molecular lines will show a flux variation with pulsation phase and time (e.g., $\left.\mathrm{H}_{2} \mathrm{O}\right)$, while other species will not (e.g., CO). These variations merely reflect the non-equilibrium chemistry that destroys and reforms molecules over a pulsation period in the shocked gas of the dust formation zone.
\end{abstract}

Key words. astrochemistry - stars: carbon - stars: AGB and post-AGB - molecular processes

\section{Introduction}

In their late stages of evolution, low-mass stars (i.e., stars with initial masses on the zero-age-main-sequence comprised between 1 and $8 M_{\odot}$ ) ascend the asymptotic giant branch (AGB) and develop cool and strong stellar winds characterised by a great variety of chemical species in the outflow detected through their ro-vibrational transitions (Olofsson 2008). With the launch of the submillimetre (submm) Herschel telescope and the beginning of science operations of the Atacama Large Millimetre Array (ALMA), our knowledge of the chemical composition of AGB winds is bound to dramatically increase with the discovery and identification of many new molecules. The wind of a AGB star develops in the dense and hot gas layers above the stellar photosphere, triggered by the formation of dust. To a first approximation, the chemical composition of the photosphere is determined by thermodynamic equilibrium (TE) owing to the high temperatures and densities of the gas (Tsuji 1973; McCabe et al. 1979). Carbon monoxide, CO, which is a very stable species, forms under TE conditions after the production of molecular hydrogen, $\mathrm{H}_{2}$. If the stellar photosphere is oxygen-rich, the excess

* Full Table A.1 is only available at the CDS via anonymous ftp to cdsarc.u-strasbg.fr $(130.79 .128 .5)$ or via

http://cdsarc.u-strasbg.fr/viz-bin/qcat?]/A+A/545/A12 oxygen not locked up in CO drives a wind chemistry dominated by oxygen-bearing molecules such as water, $\mathrm{H}_{2} \mathrm{O}$, and silicon monoxide, $\mathrm{SiO}$, and the dust forming in the wind includes silicates (e.g., forsterite $\mathrm{Mg}_{2} \mathrm{SiO}_{4}$ ) and metal oxides (e.g., alumina $\mathrm{Al}_{2} \mathrm{O}_{3}$ ). Conversely, if the star has experienced third-dredge up in the upper part of its AGB ascension, its photosphere may become carbon-rich. The chemistry of the wind then reflects the excess carbon not locked up in $\mathrm{CO}$ and is rich in $\mathrm{C}$-bearing species such as acetylene, $\mathrm{C}_{2} \mathrm{H}_{2}$, and hydrogen cyanide, $\mathrm{HCN}$. Those stars form large amounts of carbon dust close to their photosphere. This simple picture was seriously questioned by the detection at millimetre $(\mathrm{mm})$ wavelengths of $\mathrm{HCN}$ in O-rich AGB stars (Deguchi \& Goldsmith 1985). In carbon stars, SiO was observed (Olofsson et al. 1982), but because TE models of carbon stars predicted its formation, it did not come as a surprise to observe this O-bearing species in carbon-rich environments. However, the observed abundances were much higher than those derived from TE. Several mechanisms were proposed to explain these unexpected species, including an ion-molecule chemistry in the outer part of the stellar wind experiencing the penetration of the ultraviolet (UV) interstellar radiation field and cosmic rays (Nejad \& Millar 1988). The later detection by the Infrared Space Observatory, ISO, of hot vibrational transitions of carbon dioxide, $\mathrm{CO}_{2}$, in the cool supergiant NML Cyg 
(Justtanont et al. 1996) indicated that carbon-bearing species could form in the deep layers of O-rich stellar winds. The detection of water, $\mathrm{H}_{2} \mathrm{O}$, with the SWAS satellite at submm wavelength by Melnick et al. (2001) and that of hydroxyl, OH, by Ford et al. (2004) in the carbon star IRC+10216 provided additional evidence of the complex chemistry of AGB outflows, where comets (Melnick et al. 2001), grain-surface chemistry (Willacy 2004), or UV photodissociation in the intermediate envelope (Agúndez \& Cernicharo 2006) were proposed as possible sources of water.

However, that these unexpected species could form by means of some non-equilibrium chemistry in the inner wind was soon found to be viable by surveys of specific species in a large sample of objects, e.g., SiO (Schöier et al. 2006). Just above the photosphere, the gas experiences the passage of shocks triggered by the pulsation of the star and these shocked gas layers are the locus of dust formation (Bowen 1988). These dense molecular gas layers has been detected by both observations with ISO (Tsuji et al. 1997) and near-infrared (IR) interferometry (Perrin et al. 2004). A semi-analytical model for the physics of these shocked regions based on the work of Fox \& Wood (1985) and Bertschinger \& Chevalier (1985) was proposed by Cherchneff (1996) assuming that the shock energy was dissipated in the immediate postshock gas by the collisional dissociation of $\mathrm{H}_{2}$ and that subsequent cooling was provided by adiabatic expansion. Using a similar formalism for the inner wind of IRC +10216 , Willacy \& Cherchneff (1998) modelled the gas-phase chemistry and showed that the collisional destruction of $\mathrm{CO}$ in the shocks could release free atomic oxygen and trigger the formation of $\mathrm{OH}$ and $\mathrm{SiO}$. The formations of $\mathrm{HCN}, \mathrm{CO}_{2}$, and $\mathrm{CS}$ were later described in the inner wind of the O-rich Mira star IK Tau by Duari et al. (1999). These two studies therefore highlighted the importance of the shock-induced non-equilibirum chemistry in the inner wind of AGB stars in unleashing the synthesis of molecules that were not expected to form under photospheric TE conditions. More generally, Cherchneff (2006) studied the inner wind composition as a function of the carbon-to-oxygen $(\mathrm{C} / \mathrm{O})$ ratio of the stellar photosphere and confirmed the formation of O-bearing species in carbon stars and that of C-bearing molecules in O-rich AGB stars as a result of non-equilibrium chemistry induced by periodic shocks. Several observations of high energy rotational transitions of $\mathrm{HCN}, \mathrm{SiS}, \mathrm{CS}$, and $\mathrm{SiO}$ in AGBs and supergiants at $\mathrm{mm}$ and submm wavelengths corroborated these results (e.g., Schöier et al. 2006, 2007; Ziurys et al. 2007, 2009; Decin et al. 2008).

A next step in understanding the complexity of the wind chemistry close to the stellar photosphere was achieved with Herschel and the confirmation of the widespread presence of water in the dust formation zone of several carbon stars (Decin et al. 2010b, Neufeld et al. 2010, 2011a,b). Agúndez et al. (2011) proposed that the partial dissociation of ${ }^{13} \mathrm{CO}$ and $\mathrm{SiO}$ by UV photons penetrating deep inside the clumpy stellar wind could produce free atomic oxygen and lead to the subsequent formation of $\mathrm{H}_{2} \mathrm{O}$. This scenario would destroy other species in the deep layers and have some impact on the water isotopologue abundances as discussed by Neufeld et al. (2011a). Both effects have not yet been tested observationally. Updating the inner wind model for IRC+10216, Cherchneff (2011a) showed that the nonequilibrium chemistry triggered by the shocks could form water in the dust formation zone in competition with the synthesis of $\mathrm{SiO}$, with abundances that agree excellently with those derived from observations.

In this study, we present a complete, updated chemical model of the inner wind of the carbon star IRC +10216 , based on the
Table 1. Stellar parameters for IRC +10216 .

\begin{tabular}{lcc}
\hline \hline$M_{\star}\left(M_{\odot}\right)$ & 2 & 1 \\
$R_{\star}(\mathrm{cm})$ & $6.5 \times 10^{13}$ & 2 \\
$\mathrm{C} / \mathrm{O}$ & 1.4 & 3 \\
$P($ days $)$ & 650 & 4 \\
$r_{\mathrm{s}}\left(R_{\star}\right)$ & 1.2 & 2 \\
$V_{\mathrm{s}}\left(\mathrm{km} \mathrm{s}^{-1}\right)$ & 20 & 2 \\
$n_{\text {gas }}\left(r_{\mathrm{s}}\right)\left(\mathrm{cm}^{-3}\right)$ & $3.6 \times 10^{13}$ & 5 \\
$T_{\text {gas }}\left(r_{\mathrm{s}}\right)(\mathrm{K})$ & 2062 & 5 \\
\hline
\end{tabular}

References. (1) Willacy \& Cherchneff (1998); (2) Ridgway \& Keady (1988); (3) Winters et al. (1994); (4) Witteborn et al. (1980); (5) Cherchneff (2011a).

non-equilibrium chemistry approach mentioned previously. The synthesis of classical molecules including water and several new species is considered in the gas-phase, comprising hydrides, chlorine and phosphorous compounds, hydrocarbons and aromatics, and gas-phase precursors to silicon carbide and metalsulphide dust. The goal of such a study is to confirm the formation of already detected species and predict new molecules potentially observable by means of their high excitation transitions in the inner wind. In Sect. 2, we give a brief description of the physics of the shocked layers, while the chemistry is discussed in Sect. 3, the results for the various families of species are presented in Sect. 4, and a discussion follows in Sect. 5.

\section{Physics of the inner wind}

The stellar parameters used in this study for IRC+10216 are listed in Table 1. The stellar photosphere and the outer atmospheric layers above it are assumed at TE with a solar elemental composition (Asplund et al. 2009) except for carbon where the $\mathrm{C} / \mathrm{O}$ ratio was set to 1.4 (Winters et al. 1994). The shocks are assumed to form at a radius $r_{\mathrm{s}}=1.2 R_{\star}$ with a velocity $V_{\mathrm{s}}$. The TE calculations are run until $r_{\mathrm{s}}$, which is characterised by the temperature and number density listed in Table 1, and the TE abundances are used to characterise the unshocked gas. Since the preshock gas at $r_{\mathrm{s}}$ has a high molecular component, the impact of the passage of periodic shocks is modelled by considering a postshock gas that cools via both 1) the collision-induced dissociation of $\mathrm{H}_{2}$ and 2) adiabatic expansion. The shock jump in density, temperature, and velocity is described by the Rankine-Hugoniot jump conditions applied to the pre-shock gas at $r_{\mathrm{s}}$ for a shock velocity $V_{\mathrm{s}}=20 \mathrm{~km} \mathrm{~s}^{-1}$ (Ridgway \& Keady 1981). According to Fox \& Wood (1985), radiative processes do not operate in cooling the post-shock gas owing to the modest shock strength, for which the energy loss is provided by the endothermic dissociation of $\mathrm{H}_{2}$ by collisions initiated by the prevalent chemical reaction

$\mathrm{H}_{2}+\mathrm{H}_{2} \rightarrow \mathrm{H}+\mathrm{H}+\mathrm{H}_{2}$.

Reaction 1 has a reaction rate given by $k_{\text {diss }}=1.5 \times 10^{-9} \times$ $\exp (-48346 / T) \mathrm{cm}^{3} \mathrm{~s}^{-1}$, where $T$ is the gas temperature, and operates over a length $l_{\text {diss }}$ defined as

$l_{\text {diss }}=\tau_{\text {diss }} \times v_{\text {gas }}=\frac{1}{k_{\text {diss }} \times n\left(\mathrm{H}_{2}\right)} \times\left[\frac{V_{\mathrm{s}}}{N_{\text {jump }}}\right]$,

where $n\left(\mathrm{H}_{2}\right)$ is the number density of $\mathrm{H}_{2}$ in the post-shock gas, $k_{\text {diss }}$ is the rate of reaction $1, V_{\mathrm{s}}$ is the shock velocity at $r_{\mathrm{s}}$ as given in Table 1 , and $N_{\text {jump }}$ is the Rankine-Hugoniot velocity shock jump. 
Table 2. Gas parameters in the dust formation zone of IRC+10216 taken from Cherchneff (2011a).

\begin{tabular}{lccccccccc}
\hline \hline$r$ & Shock strength & \multicolumn{2}{c}{ Preshock gas } & \multicolumn{2}{c}{ Shock Front } & \multicolumn{2}{c}{ Adiabatic expansion - start } & \multicolumn{2}{c}{ Adiabatic expansion - end } \\
\hline$\left(R_{\star}\right)$ & $\left(\mathrm{km} \mathrm{s}^{-1}\right)$ & $T$ & $n_{\text {gas }}$ & $T$ & $n_{\text {gas }}$ & $T$ & $n_{\text {gas }}$ & $T$ & $n_{\text {gas }}$ \\
\hline 1.2 & 20.0 & 2062 & $3.63(13)$ & 19725 & $1.98(14)$ & 4409 & $5.97(14)$ & 1480 & $3.63(13)$ \\
1.5 & 17.9 & 1803 & $8.24(12)$ & 15922 & $4.40(13)$ & 3870 & $1.29(14)$ & 1290 & $8.24(12)$ \\
2 & 15.5 & 1517 & $1.44(12)$ & 12081 & $7.59(12)$ & 3200 & $2.14(13)$ & 1080 & $1.44(12)$ \\
2.5 & 13.9 & 1327 & $4.24(11)$ & 9779 & $2.21(12)$ & 2750 & $6.08(12)$ & 951 & $4.24(11)$ \\
3 & 12.6 & 1190 & $1.69(11)$ & 8245 & $8.73(11)$ & 2430 & $2.35(12)$ & 848 & $1.69(11)$ \\
4 & 11.0 & 1001 & $4.51(10)$ & 6284 & $2.29(11)$ & 1790 & $4.48(11)$ & 711 & $4.51(10)$ \\
5 & 9.8 & 876 & $1.79(10)$ & 5096 & $8.94(10)$ & 1550 & $1.71(11)$ & 621 & $1.79(10)$ \\
\hline
\end{tabular}

Notes. The shocks form at $r_{\mathrm{s}}=1.2 R_{\star}$. For each radius, the temperature and number density are given for the pre-shock gas, the gas after the shock front (in the collision-induced $\mathrm{H}_{2}$ dissociation zone), and the gas at the beginning and the end of the adiabatic cooling zone. The temperature $T$ is in Kelvin and the gas number density $n_{\text {gas }}$ in $\mathrm{cm}^{-3}$.

Table 3. Chemical species included in the inner wind model of IRC+10216.

\begin{tabular}{lcccccc}
\hline \hline Classical & $\mathrm{H}_{2}$ & $\mathrm{CO}$ & $\mathrm{SiO}$ & $\mathrm{HCN}$ & $\mathrm{C}_{2} \mathrm{H}_{2}$ & $\mathrm{SiS}$ \\
\hline Hydrocarbons & $\mathrm{C}_{2} \mathrm{H}_{3}$ & $\mathrm{C}_{3} \mathrm{H}_{2}$ & $\mathrm{C}_{3} \mathrm{H}_{3}$ & $\mathrm{C}_{4} \mathrm{H}_{2}$ & $\mathrm{C}_{4} \mathrm{H}_{3}$ & $\mathrm{C}_{4} \mathrm{H}_{4}$ \\
Aromatics & $\mathrm{C}_{6} \mathrm{H}_{5}$ & $\mathrm{C}_{6} \mathrm{H}_{6}$ & & & & \\
$\mathrm{C}$ & $\mathrm{CH}$ & $\mathrm{CH}_{2}$ & $\mathrm{CH}_{3}$ & $\mathrm{C}_{2} \mathrm{H}$ & $\mathrm{C}_{2}$ & $\mathrm{C}_{3}$ \\
$\mathrm{O}$ & $\mathrm{OH}$ & $\mathrm{H}_{2} \mathrm{O}$ & $\mathrm{O}_{2}$ & $\mathrm{CO}_{2}$ & $\mathrm{HCO}^{2}$ & \\
$\mathrm{~N}$ & $\mathrm{NH}$ & $\mathrm{N}_{2}$ & $\mathrm{CN}$ & $\mathrm{NO}$ & $\mathrm{NH}_{2}$ & $\mathrm{NH}_{3}$ \\
$\mathrm{P}$ & $\mathrm{PH}$ & $\mathrm{P}_{2}$ & $\mathrm{HCP}$ & $\mathrm{CP}$ & $\mathrm{PN}$ & \\
$\mathrm{Si}$ & $\mathrm{SiN}$ & $\mathrm{SiC}$ & $\mathrm{SiC}_{2}$ & $\mathrm{Si}_{2} \mathrm{C}_{2}$ & & \\
$\mathrm{~S}$ & $\mathrm{CS}$ & $\mathrm{SH}$ & $\mathrm{H}_{2} \mathrm{~S}$ & $\mathrm{SO}$ & & \\
$\mathrm{Mg}$ & $\mathrm{MgH}$ & $\mathrm{MgS}$ & $\mathrm{Mg}_{2}$ & $\mathrm{MgO}$ & & \\
$\mathrm{Fe}$ & $\mathrm{FeH}$ & $\mathrm{FeS}$ & $\mathrm{Fe}_{2}$ & $\mathrm{FeO}$ & & \\
$\mathrm{F}$ & $\mathrm{HF}$ & $\mathrm{F}_{2}$ & $\mathrm{ClF}$ & & & \\
$\mathrm{Al}$ & $\mathrm{AlH}$ & $\mathrm{AlCl}$ & & & & \\
$\mathrm{Na}$ & $\mathrm{NaH}$ & $\mathrm{NaCl}$ & & & & \\
$\mathrm{Cl}$ & $\mathrm{HCl}$ & $\mathrm{Cl}$ & & & & \\
$\mathrm{K}$ & $\mathrm{KH}$ & $\mathrm{KCl}$ & & & & \\
\hline
\end{tabular}

Once this cooling has proceeded over $l_{\text {diss }}$, we assume that later cooling occurs via adiabatic expansion. As modelled by Bertschinger \& Chevalier (1985), the fluid equations that describe the conservation of mass, momentum, and energy are parametrised and solved for the boundary conditions imposed by stellar gravity and the return of the shocked gas to its initial pre-shock position. Typical excursions of the gas layers over several pulsation periods are illustrated in Willacy \& Cherchneff (1998) and Cherchneff (2011a) for IRC+10216. The pre-shock gas temperature and density profiles are derived as a function of radius using the formalism of Cherchneff et al. (1992), where the impact of shocks on the gas density is described by an extended scale-height formalism for the initial conditions listed in Table 1. The derived pre- and post-shock gas parameters at various radius are those assumed in Cherchneff (2011a) and listed in Table 2.

\section{Chemistry of the inner wind}

New families of chemical species are considered along with the classical molecules already studied in previous models (Cherchneff 2006, 2011a). They include hydrides, halogens, in particular chlorine- and fluorine-bearing species, and finally phosphorous-bearing species. Small gas-phase molecular precursors to silicon carbide and metal-sulphide grains are also included. All species are listed in Table 3 where the largest molecule is the aromatic ring of benzene, $\mathrm{C}_{6} \mathrm{H}_{6}$. For metal hydrides, there is no available information on chemical reaction rates for the formation processes of several of them, namely $\mathrm{FeH}, \mathrm{MgH}, \mathrm{NaH}, \mathrm{KH}$, and $\mathrm{PH}$, at the high temperatures characterising the inner wind. We thus assume rates similar to those of documented reactions using the principle of isovalence as a guideline. Generally, the typical rates of the dominant formation pathways that consists of the reaction of the metal with $\mathrm{H}_{2}$ have an Arrhenius factor ranging from $10^{-12}$ and $10^{-9} \mathrm{~cm}^{3} \mathrm{~s}^{-1}$, an activation energy barrier of a few $1000 \mathrm{~K}$, and a mild temperature dependance (e..g, Cohen \& Westberg 1983).

The chemistry of halogens including chlorine- and fluorinebearing species is rather well-documented at high and intermediate temperatures. However, this is not the case for the phosphorous chemistry. Owing to the isovalence of phosphorous, $\mathrm{P}$, with nitrogen, $\mathrm{N}$, we assume for the P-bearing species chemical processes similar to those involving $\mathrm{N}$ and for which the rates are measured or calculated. Finally, the chemistry of silicon, Si, and sulphur, S, is poorly known and studied. For these elements, we restrict the chemical processes to the set of reactions for which reaction rates are documented.

All chemical pathways that lead to the formation of linear molecules, carbon chains, and aromatic rings, include neutralneutral processes such as termolecular, bimolecular, and radiative association reactions, whereas destruction is described by thermal fragmentation and neutral-neutral processes (i.e., oxidation reactions of hydrocarbons and all reverse processes of the formation reactions). No ions are considered in this chemistry because the UV stellar radiation field of IRC+10216 is too weak to foster the efficient photodissociation and ionisation of molecules. In total, 63 species were considered for a chemical network of 377 reactions. Details of all these processes are provided in the online Appendix 1 available at the CDS, which gives the reactions included in the chemical network and their reaction rates. It contains the following information: Col. 1 lists 
the reaction number, Col. 2 gives the reactants and the products, Col. 3 lists the A coefficient, Col. 4 lists the $\mathrm{n}$ factor, Col. 5 lists the activation energy in Kelvin, and Col. 6 gives the reference for the data. The major differences between the present chemical network and that used in previous studies (e.g., Willacy \& Cherchneff 1998; Cherchneff 2006) are explained in detail in the appendix of Cherchneff (2011a). In terms of formalism, three major changes are implemented. Firstly, the treatment of the reverse reaction of a specific process is changed. Several new rates have been measured in combustion and aerosol chemistry, and are now available. The calculation of the reverse rate from the equilibrium constant assuming detailed balance (i.e., Eq. (4) in Willacy \& Cherchneff 1998) can lead to erroneous values when the gas temperature and density decrease. Therefore, we prefer to directly enter the available measured or calculated rate values in the chemical network. When the information is unavailable, we make "educated" guesses depending on the type of the reaction and its endo-(exo)thermicity. This approach allows us to test a variety of reaction paths that would be closed if one were to strictly apply detailed balance considerations. Secondly, the chemistry involving atomic silicon, $\mathrm{Si}$, and Si-bearing species is restricted to reactions for which rates have been measured or calculated. Thirdly, as previously mentioned, the chemistry now describes the formation of a larger set of chemical species. This chemical scheme is used to solve a set of 63 stiff, coupled, ordinary differential equations (ODEs) at each radius of the inner wind. These coupled ODES are integrated over space (for the $\mathrm{H}_{2}$ cooling region defined by Eq. (2)) and time (for the adiabatic expansion over a pulsation period) for the radii and corresponding shock strengths of Table 2 . The post-shock abundances of the species at the end of the pulsation period and radius $r_{i}$ are used as pre-shock initial abundances for the successive radius $r_{i+1}$.

In all, the chemical network aims to provide a comprehensive and coherent chemical description of the wind, where known trends are reproduced or new trends are presented. It does not aim to give an exact description of all chemical processes in the wind, which is an impossible task to perform. Owing to the uncertainties in both our theoretical description of the inner wind and observational data analysis, we consider in the following sections that a good agreement has been reached when modelled abundances and derived values from observations differ by at most a factor of ten.

\section{Results}

Results for the dominant chemical species in the inner wind are summarised in Table 4. We list the abundance values derived by applying TE to the gas conditions met at $r_{\mathrm{s}}$, as well as the nonequilibrium abundances at $r_{\mathrm{s}}$ and $5 R_{\star}$, and abundances derived from the most recent observations. As stated before, all modelled and observational values differ by at most a factor of ten, except for PN (see Sect. 4.4). This good agreement is illustrated in Fig. 1 where we compare the modelled and observed abundances with respect to $\mathrm{H}_{2}$ at $5 R_{\star}$. Discrepancies between values derived from TE and non-equilibirum chemistry for O-bearing species are clear from inspection of Table 4. These discrepancies highlight once more the importance of the shock chemistry in the dust formation zone of AGB stars. We discuss below the results for the specific chemical families under study.

\subsection{Prevalent molecules}

A group of species dominates the molecular phase of the shocked, inner wind along with $\mathrm{H}_{2}$. It consists of $\mathrm{CO}, \mathrm{HCN}$,

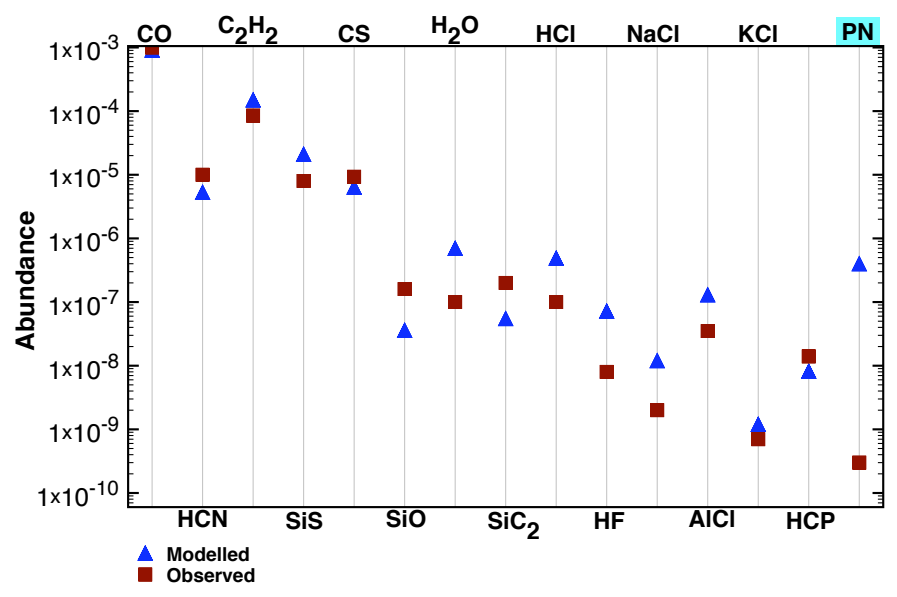

Fig. 1. Observed and modelled abundances (with respect to $\mathrm{H}_{2}$ ) for most of the molecules listed in Table 4. Abundances are at phase $\theta=1$ and $5 R_{\star}$. The agreement between modelled and observed values is satisfactory and less than a factor of ten for all species apart from PN (for CO both values overlap).

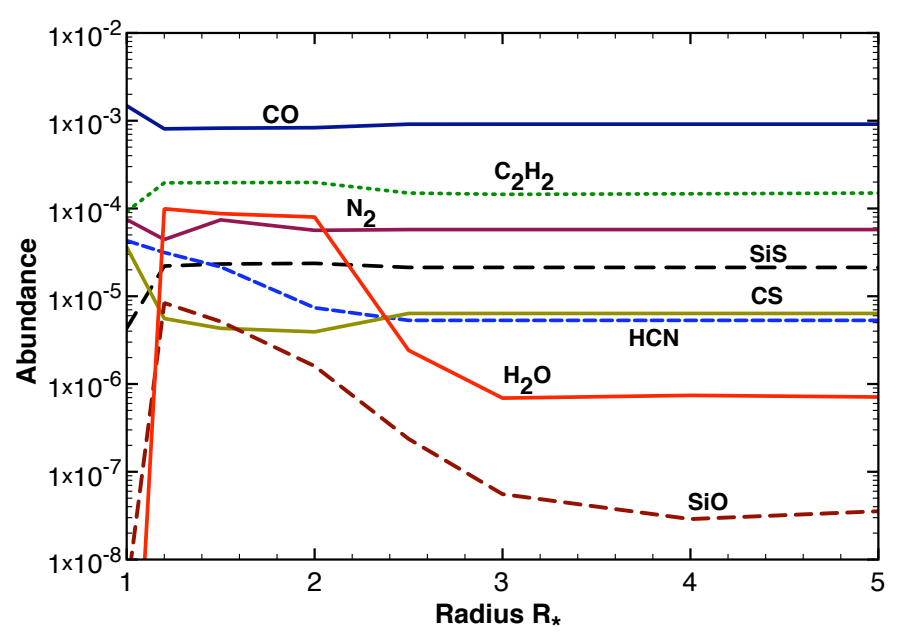

Fig. 2. Abundances (with respect to $\mathrm{H}_{2}$ ) of the prevalent molecules formed in the inner wind as a function of radius. Abundances are those at phase $\theta=1$, i.e., after one full oscillation cycle. Abundances derived by assuming thermodynamic equilibrium at $r_{\mathrm{s}}$ are moved to radius $1 R_{\star}$ for clarity.

$\mathrm{CS}, \mathrm{N}_{2}, \mathrm{C}_{2} \mathrm{H}_{2}, \mathrm{SiS}, \mathrm{SiO}$, and $\mathrm{H}_{2} \mathrm{O}$, whose abundances relative to $\mathrm{H}_{2}$ are illustrated as a function of radius in Fig. 2, while their abundances with respect to total gas and as a function of pulsation phase $\theta$ at the shock formation radius $r_{\mathrm{s}}$ are shown in Fig. 3. All molecules experience destruction in the post-shock gas at $\theta<0.05$ as seen in Fig. 3. The destruction is more or less severe depending on the species and no large discrepancies may exist between TE and non-TE abundances for specific molecules at the end of one oscillation. However, TE abundance values for several species (CS, $\mathrm{SiS}, \mathrm{SiO}$, and $\mathrm{H}_{2} \mathrm{O}$ ) differ drastically from those obtained from shock-induced, non-equilibrium chemistry, as already stressed in existing studies (Willacy \& Cherchneff 1998; Cherchneff 2006, 2011a).

The formation of O-bearing species, namely $\mathrm{H}_{2} \mathrm{O}$ and $\mathrm{SiO}$, results from the collision dissociation of $\mathrm{CO}$ in the post-shock gas. For the specific conditions of the IRC +10216 model, between $10 \%$ and $20 \%$ of $\mathrm{CO}$ molecules are destroyed at $r_{\mathrm{s}}$ in the $\mathrm{H}_{2}$ dissociation cooling part, while they quickly reform in the adiabatic expansion at phase $\theta>0.05$, making $\mathrm{CO}$ the main 
Table 4. Predicted abundances (with respect to $\mathrm{H}_{2}$ ) of selected species.

\begin{tabular}{|c|c|c|c|c|c|}
\hline Species & $\overline{\text { TE at } r_{\mathrm{s}}}$ & Predicted at $r_{\mathrm{s}}$ & Predicted at $5 R_{\star}$ & Observed & Reference \\
\hline $\mathrm{CO}$ & $1.5 \times 10^{-3}$ & $8.1 \times 10^{-4}$ & $9.1 \times 10^{-4}$ & $1.0 \times 10^{-3}$ & Herschel - Cernicharo et al. (2010a) \\
\hline $\mathrm{HCN}$ & $4.3 \times 10^{-5}$ & $3.2 \times 10^{-5}$ & $5.3 \times 10^{-6}$ & $8.0 \times 10^{-6}-2.0 \times 10^{-5}$ & IRAM - Cernicharo et al. (2011) \\
\hline $\mathrm{C}_{2} \mathrm{H}_{2}$ & $9.2 \times 10^{-5}$ & $2.0 \times 10^{-4}$ & $1.5 \times 10^{-4}$ & $8.5 \times 10^{-5}$ & TEXES/IRTF - Fonfría et al. (2008) \\
\hline $\mathrm{N}_{2}$ & $7.5 \times 10^{-5}$ & $4.4 \times 10^{-5}$ & $5.8 \times 10^{-5}$ & & \\
\hline $\mathrm{SiS}$ & $4.3 \times 10^{-6}$ & $2.2 \times 10^{-5}$ & $2.1 \times 10^{-5}$ & $2.0 \times 10^{-6}-8.0 \times 10^{-6}$ & PACS/SPIRE Herschel - Decin et al. (2010a) \\
\hline CS & $3.6 \times 10^{-5}$ & $5.6 \times 10^{-6}$ & $6.4 \times 10^{-6}$ & $9.3 \times 10^{-6}$ & SMA - Patel et al. (2009) \\
\hline $\mathrm{SiO}$ & $4.1 \times 10^{-9}$ & $8.4 \times 10^{-6}$ & $3.6 \times 10^{-8}$ & $2.0 \times 10^{-8}-3.0 \times 10^{-7}$ & PACS/SPIRE Herschel - Decin et al. (2010a) \\
\hline \multirow[t]{2}{*}{$\mathrm{H}_{2} \mathrm{O}$} & $2.0 \times 10^{-12}$ & $9.9 \times 10^{-5}$ & $7.1 \times 10^{-7}$ & $8.0 \times 10^{-8}$ & HIFI/Herschel - Neufeld et al. (2011a) \\
\hline & - & - & - & $>1 \times 10^{-7 a}$ & PACS/SPIRE Herschel - Decin et al. (2010b) \\
\hline $\mathrm{SiC}_{2}$ & $4 \times 10^{-7}$ & $3.8 \times 10^{-11}$ & $5.5 \times 10^{-8}$ & $2.0 \times 10^{-7}$ & HIFI/Herschel - Cernicharo et al. (2010a) \\
\hline $\mathrm{HCl}$ & $5.4 \times 10^{-7}$ & $6.3 \times 10^{-7}$ & $4.9 \times 10^{-7}$ & $1.0 \times 10^{-7}$ & HIFI/Herschel - Agúndez et al. (2011) \\
\hline $\mathrm{HF}$ & $1.2 \times 10^{-7}$ & $7.3 \times 10^{-8}$ & $7.2 \times 10^{-8}$ & $8.0 \times 10^{-9}$ & HIFI/Herschel - Agúndez et al. (2011) \\
\hline $\mathrm{NaCl}$ & $2.4 \times 10^{-12}$ & $6.7 \times 10^{-9}$ & $1.2 \times 10^{-8}$ & $2 \times 10^{-9}$ & SMT/ARO - Milam et al. (2007) \\
\hline $\mathrm{AlCl}$ & $1.6 \times 10^{-10}$ & $1.8 \times 10^{-10}$ & $1.3 \times 10^{-7}$ & $3.5 \times 10^{-8}$ & IRAM - Agúndez (2009) \\
\hline $\mathrm{KCl}$ & $6.4 \times 10^{-13}$ & $7.2 \times 10^{-10}$ & $1.2 \times 10^{-9}$ & $7.0 \times 10^{-10}$ & IRAM - Agúndez (2009) \\
\hline $\mathrm{AlH}$ & $3.8 \times 10^{-9}$ & $4.6 \times 10^{-6}$ & $2.0 \times 10^{-8}$ & $<1.6 \times 10^{-6 c}$ & PACS/SPIRE Herschel - Cernicharo et al. (2010b) \\
\hline $\mathrm{NaH}$ & $1.1 \times 10^{-11}$ & $1.4 \times 10^{-6}$ & $9.9 \times 10^{-12}$ & $<3.0 \times 10^{-9 c}$ & PACS/SPIRE Herschel - Cernicharo et al. (2010b) \\
\hline $\mathrm{KH}$ & $2.4 \times 10^{-12}$ & $1.1 \times 10^{-7}$ & $9.0 \times 10^{-13}$ & $<3.0 \times 10^{-9 c}$ & PACS/SPIRE Herschel - Cernicharo et al. (2010b) \\
\hline $\mathrm{MgH}$ & $3.7 \times 10^{-9}$ & $7.9 \times 10^{-9}$ & $5.2 \times 10^{-12}$ & $<4.0 \times 10^{-8 c}$ & PACS/SPIRE Herschel - Cernicharo et al. (2010b) \\
\hline $\mathrm{FeH}$ & $1.4 \times 10^{-10}$ & $6.5 \times 10^{-9}$ & $4.3 \times 10^{-12}$ & $<1.0 \times 10^{-9 c}$ & PACS/SPIRE Herschel - Cernicharo et al. (2010b) \\
\hline $\mathrm{PH}$ & $1.8 \times 10^{-9}$ & $5.6 \times 10^{-11}$ & $N$ & & \\
\hline $\mathrm{SH}$ & $1.4 \times 10^{-7}$ & $2.3 \times 10^{-12}$ & $N$ & & \\
\hline $\mathrm{NH}$ & $2.8 \times 10^{-11}$ & $2.6 \times 10^{-13}$ & $N$ & & \\
\hline \multirow[t]{2}{*}{ HCP } & $2.6 \times 10^{-7}$ & $1.0 \times 10^{-8}$ & $8.2 \times 10^{-9}$ & $1.4 \times 10^{-8 b}$ & IRAM - Agúndez et al. (2007) \\
\hline & - & - & - & $3.0 \times 10^{-8}$ & SMT/ARO - Milam et al. (2008) \\
\hline $\mathrm{PN}$ & $1.0 \times 10^{-10}$ & $4.4 \times 10^{-7}$ & $4.3 \times 10^{-7}$ & $3.0 \times 10^{-10}$ & SMT/ARO - Milam et al. (2007) \\
\hline $\mathrm{P}_{2}$ & $3.0 \times 10^{-12}$ & $1.3 \times 10^{-13}$ & $2.8 \times 10^{-9}$ & & \\
\hline $\mathrm{FeS}$ & $1.2 \times 10^{-12}$ & $1.2 \times 10^{-10}$ & $2.2 \times 10^{-9}$ & & \\
\hline $\mathrm{MgS}$ & $6.2 \times 10^{-13}$ & $1.5 \times 10^{-10}$ & $2.7 \times 10^{-9}$ & & \\
\hline
\end{tabular}

Notes. TE abundances are given at $r_{\mathrm{s}}$ and modelled abundances are listed for $r_{\mathrm{s}}$ and $5 R_{\star}$. Most recent abundances (with respect to $\mathrm{H}_{2}$ ) derived from observations are also listed when available with the corresponding reference. " $N$ " corresponds to abundances lower than $1 \times 10^{-15}$. (a) Refer to the maximum value reached by $\mathrm{H}_{2} \mathrm{O}$ abundance distribution with respect to $\mathrm{H}_{2}$, ${ }^{(b)}$ Refer to the $\mathrm{HCP}$ abundance for $r>20 R_{\star}$, ${ }^{(c)} 3 \sigma$ upper limits to abundances.

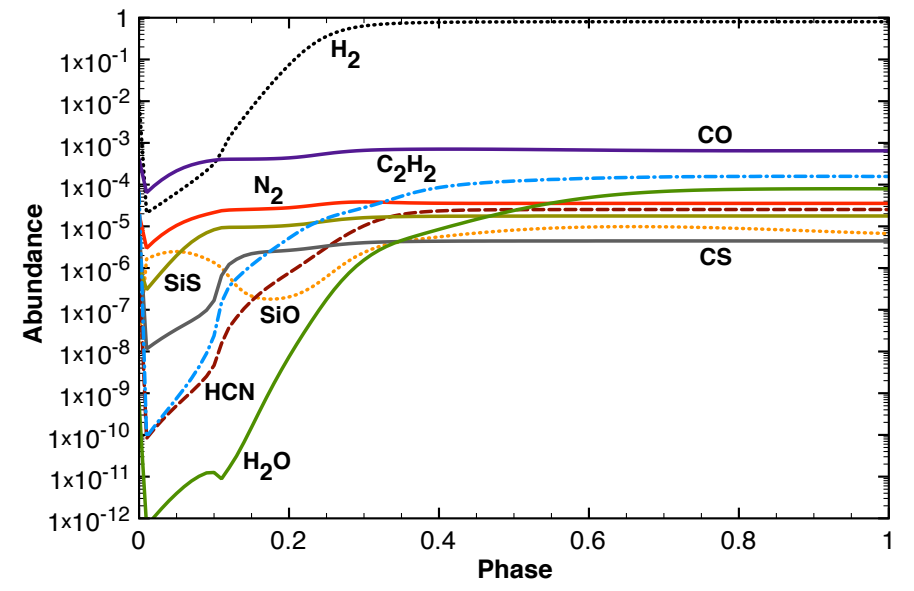

Fig. 3. Abundances (with respect to total gas) of the prevalent molecules shown in Fig. 2 as a function of phase $\theta$ at the shock formation radius $r_{\mathrm{s}}$. Destruction occurs at small values of $\theta$ but the molecules reform as the gas cools down.

provider of atomic oxygen in the post-shock gas. The formation of $\mathrm{H}_{2} \mathrm{O}$ is thus triggered by the reactions (Cherchneff 2011a)

$\mathrm{O}+\mathrm{H}_{2} \rightarrow \mathrm{OH}+\mathrm{H}$

and

$\mathrm{OH}+\mathrm{H}_{2} \rightarrow \mathrm{H}_{2} \mathrm{O}+\mathrm{H}$
The reaction given in Eq. (4) is in competition with the formation of $\mathrm{SiO}$ via the reaction

$\mathrm{Si}+\mathrm{OH} \rightarrow \mathrm{SiO}+\mathrm{H}$.

The rate of the backward process of the reaction in Eq. (5) is unknown but the reaction has an endothermicity of $\sim 40000 \mathrm{~K}$ at $2000 \mathrm{~K}$. As mentioned in Sect. 3, we assume a low rate for that reaction $\left(k=1 \times 10^{-15} \mathrm{~cm}^{3} \mathrm{~s}^{-1}\right)$ that reflects its low efficiency over the temperature range of interest. We considered the resulting water abundance for different rate values and temperature dependences. The water abundance always shows a trend similar to that reported here, i.e., a high inner value that decreases at radius $r>2 R_{\star}$ to reach a typical value of $\sim 1 \times 10^{-7}$. In contrast, the rate of the backward reaction in Eq. (5) was explicitly calculated from the equilibrium constant given in the study of Willacy \& Cherchneff (1998). This previous rate had a very low value for temperatures lower than $2000 \mathrm{~K}$, and contributed in part to the non-replenishment of $\mathrm{OH}$ and the disappearance of $\mathrm{H}_{2} \mathrm{O}$ at larger stellar radii.

The prevalent molecules in Fig. 2 come from different chemical families that are chemically linked together by the indirect key-role of the over-abundant $\mathrm{H}_{2}$ species. Molecular hydrogen is primarily involved in the formation processes of both various members of chemical families (e.g., hydrides, hydrocarbons) and specific molecules such as $\mathrm{OH}$ and $\mathrm{H}_{2} \mathrm{O}$. The destruction or formation of $\mathrm{H}_{2}$ can then impact all chemical families, which become interrelated. This is particularly true for water, owing to 


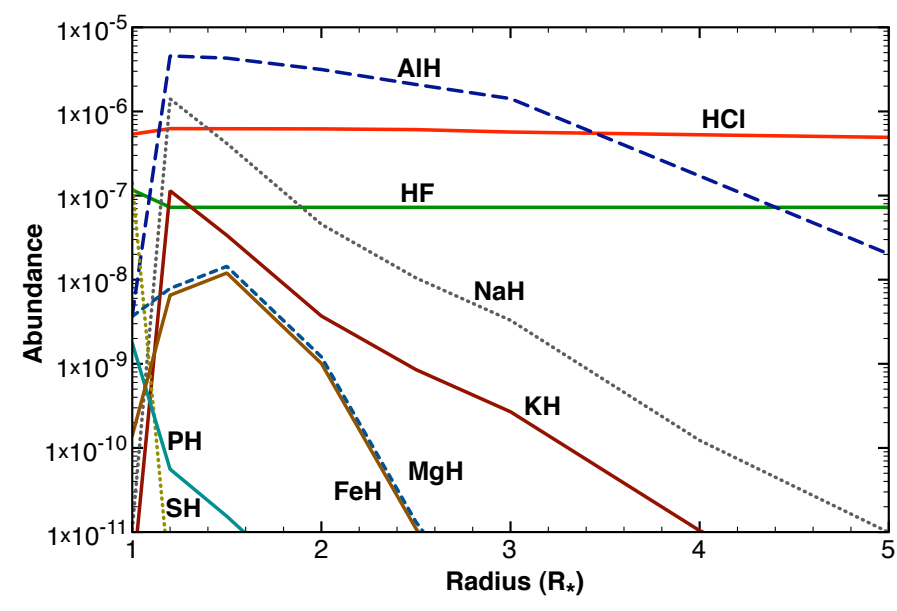

Fig. 4. Abundances (with respect to $\mathrm{H}_{2}$ ) of the hydride species formed in the inner wind as a function of radius. Abundances derived by assuming thermodynamic equilibrium at $r_{\mathrm{s}}$ are moved to radius $1 R_{\star}$ for clarity.

its link to the hydrocarbon family. The destruction of hydrocarbons, starting with $\mathrm{C}_{2} \mathrm{H}_{2}$, indeed releases $\mathrm{H}_{2}$. Through the synthesis of hydroxyl, $\mathrm{OH}$, via the reaction of atomic $\mathrm{O}$ with $\mathrm{H}_{2}$ in the post-shock gas, $\mathrm{H}_{2} \mathrm{O}$ is thus linked to the hydrocarbon family. As seen above, water is also linked to the Si-bearing species as both $\mathrm{H}_{2} \mathrm{O}$ and $\mathrm{SiO}$ species are competitors in the depletion of $\mathrm{OH}$. Atomic $\mathrm{Si}$ is also efficiently included in silicon monosulphide, $\mathrm{SiS}$, and in silicon carbide, $\mathrm{SiC}$, in the hot post-shock gas at $r_{\mathrm{s}}$. Therefore, the $\mathrm{Si}$-bearing species through their connection to $\mathrm{SiO}$ impact the water abundance, whose value listed in Table 4 is slightly higher than the value derived by Cherchneff (2011a). The chemistry of $\mathrm{SiC}$ dust precursors has been extended to the first ring $(\mathrm{SiC})_{2}$ in the present study, and as discussed in Sect. 4.6, the formation of $\mathrm{SiC}$ and the rings $\mathrm{SiC}_{2}$ and $(\mathrm{SiC})_{2}$ proceeds very early on. The more atomic $\mathrm{Si}$ is trapped in $\mathrm{SiC}$, the less $\mathrm{Si}$ is able to react with $\mathrm{OH}$ to form $\mathrm{SiO}$. Thus, the $\mathrm{SiO}$ abundance listed in Table 4 is lower than that derived by Cherchneff (2011a) by a factor of about two, resulting in a higher water abundance. However, the chemical trends and processes in both studies are alike. The formation pathways to other important molecules such as $\mathrm{C}_{2} \mathrm{H}_{2}, \mathrm{CS}$, and $\mathrm{HCN}$ were discussed in detail by Cherchneff (2006) for a carbon star with a C/O ratio equal to 1.1 and similar chemical routes operate in the inner wind of IRC+10216. Overall and as seen from Fig. 1, the most abundant species have modelled abundances that agree very well with values derived from observations.

\subsection{Hydrides}

The interest of studying light hydrides was rekindled with the launch of Herschel. The rotational spectra of these species lie in the submm and far-IR domains and are difficult to observe from Earth. The detection by HIFI onboard Herschel of the $J=1 \rightarrow$ $0,2 \rightarrow 1$ and $3 \rightarrow 2$ transitions of $\mathrm{HCl}$ and the $J=1 \rightarrow 0$ transition of HF in IRC+10216 was reported by Agúndez et al. (2011).

The gas-phase chemistry of many light hydrides has never been clearly characterised and only a few measured rates have been documented. However, their production in the laboratory from the gas phase occurs via the reaction of a metal vapour with hydrogen (Ozin \& McCaffrey 1984). For our model, we consider all known chemical reactions and extend the documented chemistry to some species according to the isovalence of specific elements (e.g., $\mathrm{N}$ and $\mathrm{P}$ ). Following the prescriptions of experimental studies, we model the formation of hydrides according to

$\mathrm{X}+\mathrm{H}_{2} \rightarrow \mathrm{XH}+\mathrm{H}$,

where $\mathrm{X}$ represents any atomic species. $\mathrm{H}_{2}$ is destroyed in the strongest shocks up to $\sim 3 R_{\star}$ but efficiently reforms in the postshock gas at phase $\theta>0.3$ (see Fig. 3). The large $\mathrm{H}_{2}$ reservoir in the gas layers insures that most hydrides are formed following the reaction in Eq. (6). The abundance variation with radius of the major hydrides is illustrated in Fig. 4. Hydrides have variations that depend on the species. The abundances of $\mathrm{AlH}$ are high out to $3 R_{\star}$ but decrease at larger radii owing to the formation of aluminium chlorine, $\mathrm{AlCl}$ (see Sect. 4.3 below). A similar behaviour applies to $\mathrm{NaH}$ and $\mathrm{KH}$ with the formation of $\mathrm{NaCl}$ and $\mathrm{KCl}$. In contrast, $\mathrm{HCl}$ and $\mathrm{HF}$ have consistently high abundances $\left(x(\mathrm{HCl}) \sim 4 \times 10^{-7}\right.$ and $\left.x(\mathrm{HF}) \sim 7 \times 10^{-8}\right)$ in the inner wind while $\mathrm{MgH}, \mathrm{FeH}, \mathrm{PH}$, and $\mathrm{SH}$ have extremely low abundances that are well below the PACS/SPIRE $3 \sigma$ detection limits estimated by Cernicharo et al. (2010b).

The Herschel detection of the $J=1 \rightarrow 0$ transition of HF by Agúndez et al. (2011) points to a constant abundance of $\sim 8 \times 10^{-9}$ with respect to $\mathrm{H}_{2}$ extending from the inner envelope up to $45 R_{\star}$. This value is lower by a factor of nine than that derived from TE in the photosphere, where most of the fluorine is in the form of HF. To reconcile these two values, they claim that $\mathrm{F}$ must be depleted on dust grains in the inner wind. Our calculated HF abundance in Table 4 has the constant value of $7.25 \times 10^{-8}$, corresponding to the solar abundance for $\mathrm{F}$. This value reflects the quick conversion of fluorine into HF by its reaction with $\mathrm{H}_{2}$ in the post-shock gas at all radii in the dust formation zone extending to $5 R_{\star}$. The result confirms that HF acts as the main reservoir of fluorine in AGB stars. The discrepancy between our kinetic results and those of Agúndez et al. is difficult to quantify. Our abundance variation through the inner wind agrees well with the abundance profile that is required to reproduce the HF HIFI data, i.e., a constant abundance distribution extending up to $45 R_{\star}$, but our calculated abundance value is higher. Agúndez et al. quote that the error in the radiative transfer model is a factor of two, while the reaction rate for the gas-phase formation of $\mathrm{HF}$ from $\mathrm{H}_{2}$ has been measured and is well-documented. The use of other available rate values does not change the present result, which indicates that fluorine is totally depleted into hydrogen fluoride in the dust formation zone.

\subsection{Chlorines}

Chlorine $(\mathrm{Cl})$ has a solar abundance with respect to hydrogen of $\sim 3.2 \times 10^{-7}$ (Asplund et al. 2009), and is present at TE in the photosphere in the form of both $\mathrm{HCl}$ and $\mathrm{Cl}$ of comparable abundances with respect to $\mathrm{H}_{2}$ of $\sim 2.6 \times 10^{-7}$. Chlorine-bearing species have long been observed in circumstellar envelopes of AGB stars. Recent large molecular surveys confirm the presence of $\mathrm{NaCl}, \mathrm{AlCl}$, and $\mathrm{KCL}$ in IRC+10216 (Tenenbaum et al. 2010) when the hydride $\mathrm{HCl}$ was detected with Herschel and unambiguously found very close to the star (see Sect. 4.2). Shinnaga et al. (2009) also identified $\mathrm{KCl}$ lines in their e-SMA observations that have a very compact distribution centred on the star. Results for the prevalent Cl-bearing species are shown in Fig. 5. Clearly, $\mathrm{HCl}$ is still the dominant $\mathrm{Cl}$-bearing species in the dust formation zone, and readily forms at $r_{\mathrm{s}}$ with an almost constant abundance throughout the inner wind and a value of $3.9 \times 10^{-7}$ at $5 R_{\star}$. This values perfectly agrees with that derived by Agúndez et al. (2011) and is very close to the TE abundance 


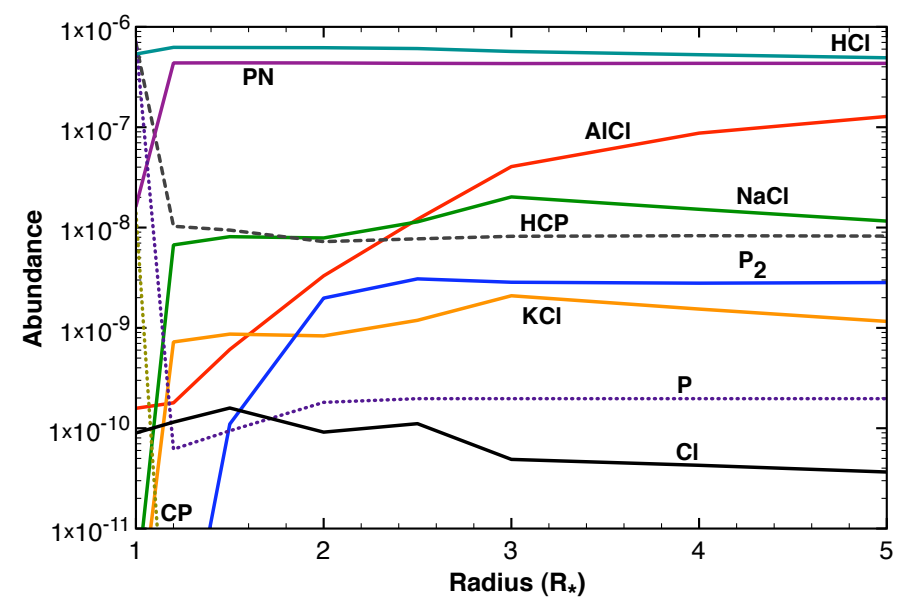

Fig. 5. Abundances (with respect to $\mathrm{H}_{2}$ ) of chlorine- and phosphorusbearing species as a function of radius. Abundances derived by assuming thermodynamic equilibrium at $r_{\mathrm{s}}$ are moved to radius $1 R_{\star}$ for clarity.

of $\mathrm{HCl}$ at $r_{\mathrm{s}}$. However, $\mathrm{HCl}$ is destroyed in the shock at $r_{\mathrm{s}}$ and reforms in the post-shock region at phase $\theta \geq 0.2$ to finally reach an abundance after one oscillation very close to the TE value. Other Cl-bearing species, namely $\mathrm{AlCl}, \mathrm{NaCl}$, and $\mathrm{KCl}$ in decreasing order of importance, are also present in the wind and their production originates directly from that of $\mathrm{HCl}$ via the reaction

$\mathrm{X}+\mathrm{HCl} \rightarrow \mathrm{XCl}+\mathrm{H}$,

where $X$ is the metal. All documented reactions of Eq. (7) are fast, with activation energy barriers of a few thousand degrees (e.g., Husain \& Marshall 1986), requiring high temperatures to proceed.

Very much like $\mathrm{H}_{2}$ for hydrides, $\mathrm{HCl}$ acts as the production agent of $\mathrm{Cl}$-bearing species in the dust formation zone of AGB stars. The chemistry of chlorine depends essentially on the hydrogen and the chlorine content of the gas through $\mathrm{HCl}$, and is thus independent of the $\mathrm{C} / \mathrm{O}$ ratio of the photosphere. Since $\mathrm{HCl}$ is a rather stable molecule (with a dissociation energy $D_{0}=4.4 \mathrm{eV}$ ), it should also form efficiently in oxygen-rich AGB stars and by a chemical pathway similar to the reaction in Eq. (6) owing to the large $\mathrm{H}_{2}$ reservoir of AGB winds. Other Cl-bearing species will also form via the reaction in Eq. (7) provided that metal atoms are available in the gas phase. $\mathrm{HCl}$ and other Cl-bearing species are thus expected to be present with intermediate-to-high abundances in the inner wind of oxygenrich $\mathrm{AGB}$ stars. The detection of high energy transitions of $\mathrm{NaCl}$ towards the O-rich supergiant VY CMa and the O-rich Mira IK Tau by Milam et al. (2007) indeed indicates that these two species form close to the star, with abundances of $5 \times 10^{-9}$ and $4 \times 10^{-9}$, respectively. Our results corroborate these observations and we predict that $\mathrm{HCl}, \mathrm{NaCl}$, and $\mathrm{KCl}$ should be observable in the dust formation zone of O-rich evolved stars. The abundance of $\mathrm{AlCl}$ may be lower because 1) the Al-bearing species $\mathrm{AlOH}$ has a high abundance in the wind acceleration zone - Tenenbaum \& Ziurys (2010) derived a AlOH abundance of $\sim 1 \times 10^{-7}$ for VY Cma - and 2) a large fraction of $\mathrm{Al}$ is expected to be depleted in alumina, $\mathrm{Al}_{2} \mathrm{O}_{3}$, in the stellar wind. In reality, $\mathrm{AlCl}$ in VY CMa was not detected by Tenenbaum \& Ziurys (2010).

\subsection{Phosphorous bearing species}

The phosphorous-bearing molecules $\mathrm{HCP}, \mathrm{CP}, \mathrm{C}_{2} \mathrm{P}$, and $\mathrm{PN}$ have been detected in the wind of IRC+10216 (Guélin et al. 1990, Agúndez et al. 2007; Milam et al. 2008; Halfen et al. 2008; He et al. 2008; Tenenbaum et al. 2010). While the shapes of the $\mathrm{C}_{2} \mathrm{P}$ and $\mathrm{CP}$ line profiles are indicative of a shell-like distribution with a formation locus in the outer envelope induced by UV photodissociation, HCP and PN have been found close to the star. The chemistry of phosphorous is poorly documented and we use the isovalence of $\mathrm{P}$ with $\mathrm{N}$ to estimate the rates of a set of basic formation and destruction processes derived from the equivalent processes involving $\mathrm{N}$. We consider a few molecules including $\mathrm{PN}, \mathrm{HCP}, \mathrm{CP}$, and $\mathrm{P}_{2}$. The latter species was chosen to reflect the refractory nature of phosphorous and its ability to form clusters. Results for P-bearing molecules are shown in Fig. 5. PN is the prevalent phosphorous compound followed by HCP and $\mathrm{P}_{2}$. PN is chiefly formed at $r_{\mathrm{s}}$ by the two reactions

$\mathrm{N}+\mathrm{CP} \rightarrow \mathrm{PN}+\mathrm{C}$

and

$\mathrm{CN}+\mathrm{CP} \rightarrow \mathrm{PN}+\mathrm{C}_{2}$.

Similar reactions for the isovalent element $\mathrm{N}$ have measured rates at high temperatures. The resulting abundances for PN are quite high $\left(\sim 3 \times 10^{-7}\right)$ through the inner wind with a rapid formation at $r_{\mathrm{s}}$ where the molecule reaches its final abundance at phase $\theta=0.5$ in the post-shock excursion. This is coherent with the fact that PN mimics $\mathrm{N}_{2}$ which shows a similar behaviour: a rapid synthesis in the post-shock gas at $r_{\mathrm{s}}$ and a constant high value across the inner wind (see Fig. 2). At $r_{\mathrm{s}}$ and at the early phases of the post-shock adiabatic excursion, phosphorous is quickly integrated into $\mathrm{CP}$ which later distributes $\mathrm{P}$ into $\mathrm{HCP}$ and PN. Milam et al. (2008) observed several rotational lines of PN and HCP in the inner wind of IRC+10216 and derived a low PN abundance of $\sim 3 \times 10^{-10}$. Our modelled value is greater by a factor of 1000 . However, our derived HCP abundance of $8.2 \times 10^{-9}$ agrees well with the observations by Agúndez et al. (2007) and Milam et al. (2008). The former study derived an abundance value of $1.4 \times 10^{-8}$ for radii larger than $20 R_{\star}$, and claimed that a depletion onto dust grains is necessary to reconcile the high abundances derived assuming TE in the dust formation region with those at $20 R_{\star}$. The present results indicate that HCP depletion in dust may not be necessary because the high TE abundances at $r<r_{\mathrm{s}}\left(\sim 3.0 \times 10^{-7}\right)$ quickly drop to $8.2 \times 10^{-9}$ at larger radii owing to the non-equilibrium chemistry and the partial conversion of $\mathrm{P}$ into $\mathrm{CP}$ and $\mathrm{PN}$ in the post-shock gas.

The high abundances of PN obtained in the model compared to observations force us to test the P chemistry and assess the conditions for which the conversion of $\mathrm{CP}$ into HCP and PN is effective. We decrease all chemical rates by a factor of ten but have no success in diminishing the PN abundance in the inner wind. We also lower the rates of the two forward reactions given in Eqs. (8) and (9), by a factor of 100 , which results in decreasing the PN abundance to $\sim 3.5 \times 10^{-8}$ but in increasing the HCP abundances to $3.2 \times 10^{-7}$, which is high to agree with observations. The assumption of isovalence between $\mathrm{P}$ and $\mathrm{N}$ requires a phosphorous chemistry in which HCP and PN mimic HCN and $\mathrm{N}_{2}$. In the inner wind, nitrogen is distributed between these two species in almost equal amounts, a result corroborated by the excellent agreement of $\mathrm{HCN}$ abundances with values derived from observations. In the case of $\mathrm{P}$, this distribution is not observed and the low PN abundance derived by Milam et al. (2008) indicates a low efficiency channel for the conversion of CP into PN 


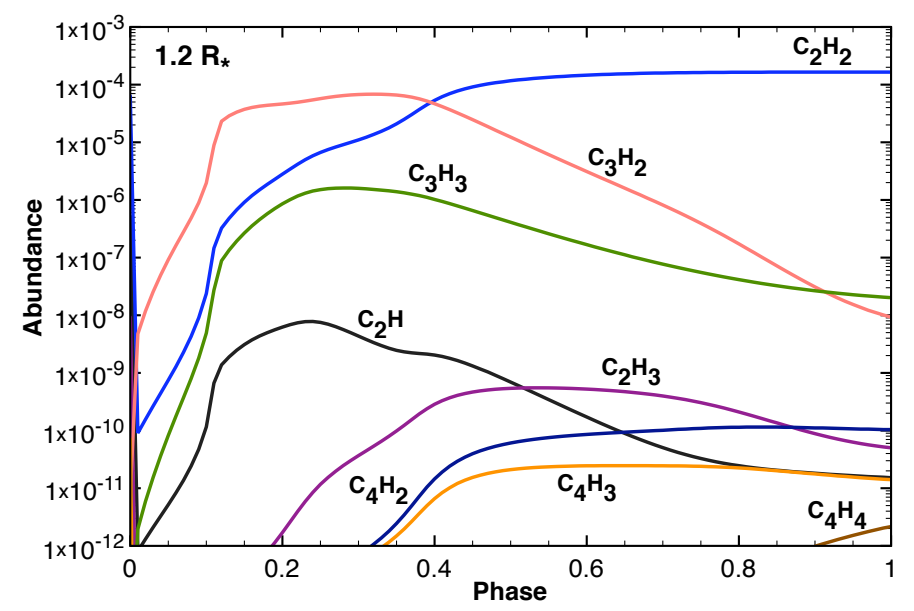

Fig. 6. Abundances (with respect to $\mathrm{H}_{2}$ ) of hydrocarbon species as a function of pulsation phase $\theta$ for the $20 \mathrm{~km} \mathrm{~s}^{-1}$ at $r_{\mathrm{s}}=1.2 R_{\star}$. No aromatic forms at these small radii owing to the presence of the oxidation agents $\mathrm{H}_{2} \mathrm{O}$ and $\mathrm{OH}$.

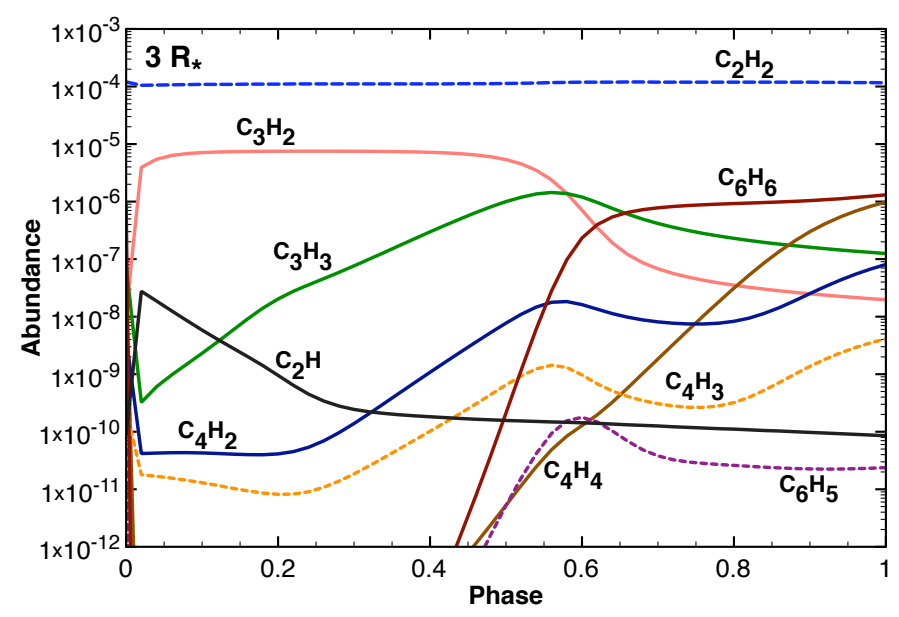

Fig. 7. Abundances (with respect to $\mathrm{H}_{2}$ ) of hydrocarbon and aromatic species as a function of pulsation phase $\theta$ for the $12.6 \mathrm{~km} \mathrm{~s}^{-1}$ at $3 R_{\star}$. Benzene starts forming at the low gas temperatures $(\sim 1000 \mathrm{~K})$ encountered at $\theta \geq 0.4$.

in the dust formation zone. According to this result, they deduce that if $\mathrm{PN} / \mathrm{N}_{2}$ were approximately equal to $\mathrm{P} / \mathrm{N}$, the $\mathrm{N}_{2}$ abundance would be very low $\left(\sim 1 \times 10^{-7}\right)$. Such a low value contradicts the high $\mathrm{N}_{2}$ abundance given by TE in the photosphere and fostered by the non-equilibrium chemistry of the inner wind (see Table 4). The discrepancy regarding the abundances of PN questions the validity of the assumption of isovalence in the case of phosphorous, highlights the different chemical processes that may control the phosphorous chemical family, and points to the need for more high resolution observations of high energy transitions of PN in the inner wind.

Finally, inspection of Fig. 5 reveals the presence of a modest amount of phosphorous dimers, $\mathrm{P}_{2}$, synthesized in the inner wind with $x\left(\mathrm{P}_{2}\right) \sim 3 \times 10^{-9}$ at $5 R_{\star}$. As $\mathrm{P}$ has even lower abundances than $\mathrm{P}_{2}$, a small population of $\mathrm{P}$ clusters may grow from $\mathrm{P}_{2}$ coalescence but the aggregation process will terminate with the formation of the stable tetrahedral $\mathrm{P}_{4}$ cluster, as observed in laser ablation of red phosphorous crystals (Bulgakov al. 2002). At most, a $\mathrm{P}_{4}$ abundance of $\sim 1 \times 10^{-10}$ may form with a leftover population of dimers with abundances $\sim 1 \times 10^{-9}$. Therefore, phosphorous clusters should not be prevalent condensates in the dust formation zone of carbon stars.

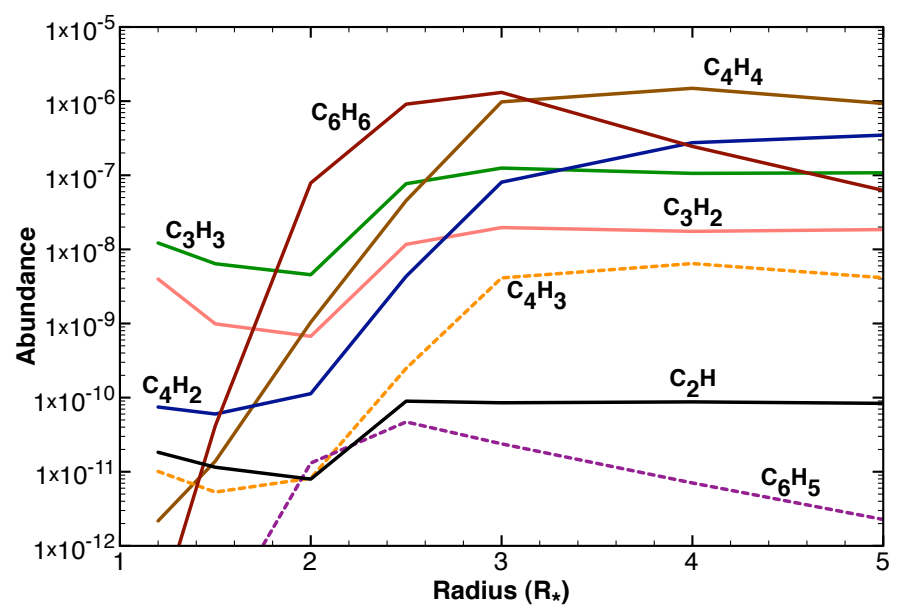

Fig. 8. Abundances (with respect to $\mathrm{H}_{2}$ ) of hydrocarbon and aromatic species as a function of radius, starting at $r_{\mathrm{s}}=1.2 R_{\star}$. Benzene starts forming at $r>2$ reaching a peak abundance value at $3 R_{\star}$. Hydrocarbons such as $\mathrm{C}_{4} \mathrm{H}_{4}, \mathrm{C}_{4} \mathrm{H}_{2}$, and propargyl $\mathrm{C}_{3} \mathrm{H}_{3}$ are also abundant in the dust formation zone.

\subsection{Carbon dust precursors: hydrocarbons and aromatics}

The formation of the first aromatic ring of benzene represents a bottleneck to the formation of polycyclic aromatic hydrocarbon (PAH) species and their growth. In the chemical scheme, it is described by the recombination of two propargyl radicals, $\mathrm{C}_{3} \mathrm{H}_{3}$ which is the dominant closure pathway, and the reaction of buten-3-ynyl radicals, $\mathrm{C}_{4} \mathrm{H}_{3}$, with $\mathrm{C}_{2} \mathrm{H}_{2}$. These two routes are the prevalent channels to aromatic formation in sooting flames on Earth (Cherchneff 2011b). The formation of $\mathrm{C}_{3} \mathrm{H}_{3}$ results from the reaction of $\mathrm{C}_{2} \mathrm{H}_{2}$ with methylene, $\mathrm{CH}_{2}$, in the immediate post-shock region at $r_{\mathrm{s}}$. Abundances with respect to $\mathrm{H}_{2}$ are shown in Fig. 6 for the $20 \mathrm{~km} \mathrm{~s}^{-1}$ shock at $r_{\mathrm{s}}$. At the high post-shock gas temperatures, only stable hydrocarbons such as $\mathrm{C}_{2} \mathrm{H}_{2}, \mathrm{C}_{3} \mathrm{H}_{2}$, and $\mathrm{C}_{3} \mathrm{H}_{3}$ can form in large amounts. In Fig. 7, the abundances for similar species are shown for a shock strength of $12.6 \mathrm{~km} \mathrm{~s}^{-1}$ at $3 R_{\star}$, with the appearance of $\mathrm{C}_{6} \mathrm{H}_{6}$ at phases $>0.4$. As apparent in Fig. 2, once $\mathrm{C}_{2} \mathrm{H}_{2}$ forms at $r_{\mathrm{s}}$, it stays abundant over the inner wind region, providing a large reservoir to grow hydrocarbons, specifically $\mathrm{C}_{3} \mathrm{H}_{2}$. Once $\mathrm{C}_{3} \mathrm{H}_{3}$ starts to form from the reaction of $\mathrm{C}_{3} \mathrm{H}_{2}$ with $\mathrm{H}_{2}, \mathrm{C}_{6} \mathrm{H}_{6}$ quickly builds up when the gas temperature drops. Indeed, higher temperature values favour the reverse routes to the formation of $\mathrm{C}_{6} \mathrm{H}_{6}$, which are endothermic channels. The clincher to build up the $\mathrm{C}_{6} \mathrm{H}_{6}$ ring are thus the lower gas temperatures encountered at phases $>0.4$. The abundances of hydrocarbons and aromatics as a function of radius are illustrated in Fig. 8. The formation of aromatics is delayed to $r \geq 2.5 R_{\star}$ because oxygen-bearing species are present at smaller radii. Water and hydroxyl are the main oxidation agents to hydrocarbons and aromatics in the gas, and $\mathrm{C}_{6} \mathrm{H}_{6}$ forms when the O-bearing species abundances drop at $r \geq 2.5 R_{\star}$ (see Fig. 2). According to Fig. 8, the prevalent hydrocarbon species that escape the inner envelope are $\mathrm{C}_{4} \mathrm{H}_{4}, \mathrm{C}_{4} \mathrm{H}_{2}$, and $\mathrm{C}_{3} \mathrm{H}_{3}$, although the growth and condensation of aromatics to amorphous carbon (AC) grains may alter this result to some extent.

Central to this study is the formation of the first aromatic ring as a bottleneck process to AC dust synthesis in carbon stars. As seen in Fig. 8. the reported abundance of $\mathrm{C}_{6} \mathrm{H}_{6}$ gradually increases with stellar radius up to $\sim 3 R_{\star}$, where it reaches a maximum value to gradually decrease up to $5 R_{\star}$. This result points to a specific radius range where AC dust has a chance to efficiently grow provided that the growing agent, in this case 
I. Cherchneff: The dust formation zone of IRC+10216 revisited

Table 5. Derived dust-to-gas mass ratio as a function of radius in the dust formation zone of IRC+10216.

\begin{tabular}{lccccccc}
\hline \hline$r\left(R_{\star}\right)$ & 1.2 & 1.5 & 2 & $\mathbf{2 . 5}$ & $\mathbf{3}$ & $\mathbf{4}$ & 5 \\
\hline$n(\text { gas })^{a}$ & $3.65(13)$ & $8.20(12)$ & $1.43(12)$ & $\mathbf{4 . 2 2}(\mathbf{1 1})$ & $\mathbf{1 . 6 6}(\mathbf{1 1})$ & $\mathbf{4 . 4 7}(\mathbf{1 0})$ & $1.74(10)$ \\
$x\left(\mathrm{C}_{2} \mathrm{H}_{2}\right)$ & $1.65(-4)$ & $1.53(-4)$ & $1.51(-4)$ & $\mathbf{1 . 2 0}(-\mathbf{4})$ & $\mathbf{1 . 1 6}(-\mathbf{4})$ & $\mathbf{1 . 1 8}(-\mathbf{4})$ & $1.20(-4)$ \\
$x\left(\mathrm{C}_{6} \mathrm{H}_{6}\right)$ & $1.05(-13)$ & $4.38(-11)$ & $9.70(-8)$ & $\mathbf{9 . 1 0}(-\mathbf{7})$ & $\mathbf{1 . 3 1}(-\mathbf{6})$ & $\mathbf{2 . 4 5}(-\mathbf{7})$ & $6.25(-8)$ \\
$N_{\text {tot }}\left(\mathrm{C}_{2} \mathrm{H}_{2}\right)$ & $5.04(51)$ & $3.43(51)$ & $1.74(51)$ & $\mathbf{8 . 5 2 ( 5 0 )}$ & $\mathbf{5 . 7 6 ( 5 0 )}$ & $\mathbf{3 . 8 2}(\mathbf{5 0})$ & $2.98(50)$ \\
$N_{\text {tot }}\left(\mathrm{C}_{6} \mathrm{H}_{6}\right)$ & $3.21(42)$ & $9.81(44)$ & $1.12(48)$ & $\mathbf{6 . 4 6}(\mathbf{4 8})$ & $\mathbf{6 . 5 0}(\mathbf{4 8})$ & $\mathbf{7 . 9 4}(\mathbf{4 7})$ & $1.55(47)$ \\
$Y\left(\mathrm{C}_{6} \mathrm{H}_{6}\right)$ & 0 & 0 & $0.2 \%$ & $\mathbf{2 . 7 \%}$ & $\mathbf{3 . 3 8 \%}$ & $\mathbf{0 . 6 \%}$ & $0.1 \%$ \\
$m\left(\mathrm{C}_{24} \mathrm{H}_{12}\right)^{a}$ & $1.60(21)$ & $4.89(23)$ & $5.56(26)$ & $\mathbf{3 . 2 2}(\mathbf{2 7})$ & $\mathbf{3 . 2 4}(\mathbf{2 7})$ & $\mathbf{3 . 9 5}(\mathbf{2 6})$ & $7.73(25)$ \\
$N_{\text {growth }}\left(\mathrm{C}_{2} \mathrm{H}_{2}\right)$ & $2.89(43)$ & $8.83(45)$ & $1.01(49)$ & $\mathbf{5 . 8 1}(\mathbf{4 9})$ & $\mathbf{5 . 8 5}(\mathbf{4 9 )}$ & $\mathbf{7 . 1 4}(\mathbf{4 8})$ & $1.40(48)$ \\
$N_{\text {growth }}\left(\mathrm{C}_{2} \mathrm{H}_{2}\right) / N_{\text {tot }}\left(\mathrm{C}_{2} \mathrm{H}_{2}\right)$ & $0 \%$ & $0.0003 \%$ & $0.58 \%$ & $\mathbf{6 . 8 3 \%}$ & $\mathbf{1 0 . 1 6 \%}$ & $\mathbf{1 . 8 7 \%}$ & $0.47 \%$ \\
$m(\text { AC dust })^{a}$ & $1.60(21)$ & $4.89(23)$ & $\mathbf{5 . 5 6}(\mathbf{2 6})$ & $\mathbf{3 . 2 2}(\mathbf{2 7})$ & $\mathbf{3 . 2 4}(\mathbf{2 7})$ & $\mathbf{3 . 9 5}(\mathbf{2 6})$ & $7.73(25)$ \\
$m$ (dust $) / m(\text { gas })^{b}$ & & & $1.30 \times 10^{-5}$ & $\mathbf{1 . 2 2} \times \mathbf{1 0}^{-\mathbf{4}}$ & $\mathbf{1 . 7 5} \times \mathbf{1 0}^{-\mathbf{4}}$ & $\mathbf{3 . 2 8} \times \mathbf{1 0}$ & $8.37 \times 10^{-6}$ \\
\hline
\end{tabular}

Notes. We list the pre-shock gas number density, acetylene and benzene abundances, the total number of $\mathrm{C}_{2} \mathrm{H}_{2}$ and $\mathrm{C}_{6} \mathrm{H}_{6}$ molecules, the $\mathrm{C}_{6} \mathrm{H}_{6}$ yield ( $\equiv$ number of $\mathrm{C}$ atoms initially in $\mathrm{C}_{2} \mathrm{H}_{2}$ locked into $\mathrm{C}_{6} \mathrm{H}_{6}$ ), the mass of coronene $\mathrm{C}_{24} \mathrm{H}_{12}$, the total number of $\mathrm{C}_{2} \mathrm{H}_{2}$ molecules depleted in the growth from $\mathrm{C}_{6} \mathrm{H}_{6}$ to $\mathrm{C}_{24} \mathrm{H}_{12}$, the corresponding number fraction of $\mathrm{C}_{2} \mathrm{H}_{2}$ depleted in the growth from $\mathrm{C}_{6} \mathrm{H}_{6}$ to $\mathrm{C}_{24} \mathrm{H}_{12}$ in $\%$, and the mass of $\mathrm{AC}$ dust formed (see text for detail). The region of efficient $\mathrm{AC}$ dust formation corresponds to numbers in boldface. ${ }^{(a)} \mathrm{Gas}^{-3}$ number densities are in $\mathrm{cm}^{-3}$ and masses in g. ${ }^{(b)}$ The estimated total dust-to-gas mass ratio ranges from $1.2 \times 10^{-3}$ to $5.8 \times 10^{-3}$ (see text for more detail).

acetylene, $\mathrm{C}_{2} \mathrm{H}_{2}$, is still abundant in the gas phase. To test our results, we assess the mass of $\mathrm{AC}$ dust that can grow from the $\mathrm{C}_{6} \mathrm{H}_{6}$ and $\mathrm{C}_{2} \mathrm{H}_{2}$ masses in the radius range of interest, compare the derived $\mathrm{AC}$ mass to the gas mass, and derive dust-to-gas mass ratios. It has been found that $\mathrm{IRC}+10216$ is heavily dust-rich and has a dust-to-gas mass ratio comprised between $1.4 \times 10^{-3}$ and $4 \times 10^{-3}$ based on various studies (e.g., Ivezić \& Elitzur 1996; Groenewegen 1998; Decin et al. 2010a). In Table 5, we list the abundances and total masses of $\mathrm{C}_{2} \mathrm{H}_{2}$ and $\mathrm{C}_{6} \mathrm{H}_{6}$ formed as a function of radius. Spherical symmetry for the inner wind is assumed to calculate the shell volume and the total number of $\mathrm{C}_{2} \mathrm{H}_{2}$ and $\mathrm{C}_{6} \mathrm{H}_{6}$ molecules. We assume that the growth of $\mathrm{C}_{6} \mathrm{H}_{6}$ to coronene, $\mathrm{C}_{24} \mathrm{H}_{12}$, proceeds through the H-abstraction$\mathrm{C}_{2} \mathrm{H}_{2}$-addition (HACA) mechanism (Frenklach et al. 1984) with the addition of nine $\mathrm{C}_{2} \mathrm{H}_{2}$ molecules, and that once coronene forms, the growth of grains continues via $\mathrm{C}_{24} \mathrm{H}_{12}$ dimerisation, coalescence, and coagulation (Cherchneff 2011b), We discard other growth mechanisms such as the polimerisation of polyynes on aromatic radical sites proposed by Krestinin et al. (2000) because the abundances of $\mathrm{C}_{4} \mathrm{H}_{2}$ are far too low to foster a significant growth of aromatic structures through this channel. Further growth via $\mathrm{C}_{2} \mathrm{H}_{2}$ addition on the surface of grains may occur but is not considered in our simple estimate. Table 5 indicates that there exists a region of high dust-formation efficiency between $2.5 R_{\star}$ and $4 R_{\star}$. This region is characterised by high gas densities and low enough temperatures (see Table 2) to initiate aromatic growth, as illustrated in Fig. 8. Furthermore, the concentration of $\mathrm{C}_{2} \mathrm{H}_{2}$ is sufficiently high to secure growth up to $\mathrm{C}_{24} \mathrm{H}_{12}$ without depleting the $\mathrm{C}_{2} \mathrm{H}_{2}$ content too much, as illustrated in Table 5, where $\sim 10 \%$ of the $\mathrm{C}_{2} \mathrm{H}_{2}$ species are consumed in the growth to $\mathrm{C}_{24} \mathrm{H}_{12}$ at $3 R_{\star}$.

To estimate the total amount of AC dust mass formed in the inner wind and ejected at $5 R_{\star}$, we consider a simple formalism whereby a parcel of gas moves gradually from $2 R_{\star}$ to $5 R_{\star}$ over a certain time span. Assuming a microturbulent velocity of between 1 and $5 \mathrm{~km} \mathrm{~s}^{-1}$, a range characteristic of the inner wind before gas drag and acceleration by dust (Keady et al. 1988), and the stellar pulsation period of Table 1, 43 pulsations (and shocks) are necessary for that parcel to reach $5 R_{\star}$ assuming a microturbulent velocity of $1 \mathrm{~km} \mathrm{~s}^{-1}$, when the pulsation number drops to 9 for a microturbulent velocity of $5 \mathrm{~km} \mathrm{~s}^{-1}$. For the modest shock velocities and moderate post-shock conditions found in the inner wind of IRC+10216, we assume that the AC dust is not destroyed in the hot post-shock gas at each shock passage, while PAH species are, but reform in the adiabatic expansion phase. Therefore, to estimate the total AC dust mass that possibly forms in the inner wind and is ejected at $5 R_{\star}$, we assume that the total $\mathrm{C}_{24} \mathrm{H}_{12}$ mass is converted into AC grain mass and we sum up the derived masses over the number of pulsations required to reach $5 R_{\star}$, interpolating mass values from the data given in Table 5 . We obtain a total dust-to-gas mass ratio that spans the range $1.2 \times 10^{-3}-5.8 \times 10^{-3}$. When compared to values derived from observations $\left(1 \times 10^{-3}\right.$ to $\left.4 \times 10^{-3}\right)$, these numbers are satisfactory and point to a specific region in the inner wind where carbon dust grows from PAHs and graphene sheets. Our simple derivation is based on a $100 \%$ growth efficiency of $\mathrm{C}_{6} \mathrm{H}_{6}$ to $\mathrm{C}_{24} \mathrm{H}_{12}$ and a $100 \%$ conversion efficiency of $\mathrm{C}_{24} \mathrm{H}_{12}$ in $\mathrm{AC}$ dust grains. These assumptions clearly maximise the AC dust mass value at $5 R_{\star}$. However, owing to the large $\mathrm{C}_{2} \mathrm{H}_{2}$ reservoir available in this region, an additional growth process that is not considered in the present derivation includes the addition of $\mathrm{C}_{2} \mathrm{H}_{2}$ molecules at the surface of graphene sheets, a mechanism that would add mass to the final carbon dust budget of the inner wind. Without pointing to a specific value for the dust-to-gas mass ratio, our derived ratios closely agree with the dust-to-gas mass ratios derived from observations and are indicative that $\mathrm{AC}$ dust formation at these specific radii efficiently proceeds in the inner wind of IRC +10216 .

\subsection{Other dust precursors: carbides and sulphides}

\subsection{1. $\mathrm{SiC}_{2}$ and $\mathrm{SiC}$}

Silicon carbide (SiC) dust has long been observed in the wind of carbon stars through its transition at $11 \mu \mathrm{m}$ (Treffers \& Cohen 1974; Speck et al. 1997) and studies on meteorites have confirmed a AGB origin for some of the pre-solar SiC inclusions (Zinner 2007). In the laboratory, the synthesis of $\mathrm{SiC}$ nanoparticles is produced using various experimental methods (e.g., laser-induced pyrolysis of gas-phase mixtures of silane, $\mathrm{SiH}_{4}$, and hydrocarbons) and $\mathrm{SiC}$ grains are observed to form in the temperature range 1600-2000 K. These temperatures are encountered in the inner wind, and support the hypothesis that $\mathrm{SiC}$ forms from the gas phase by chemical kinetic processes similar to those active in $\mathrm{SiC}$ synthesis in the laboratory. Despite the nucleation processes not being fully understood and 


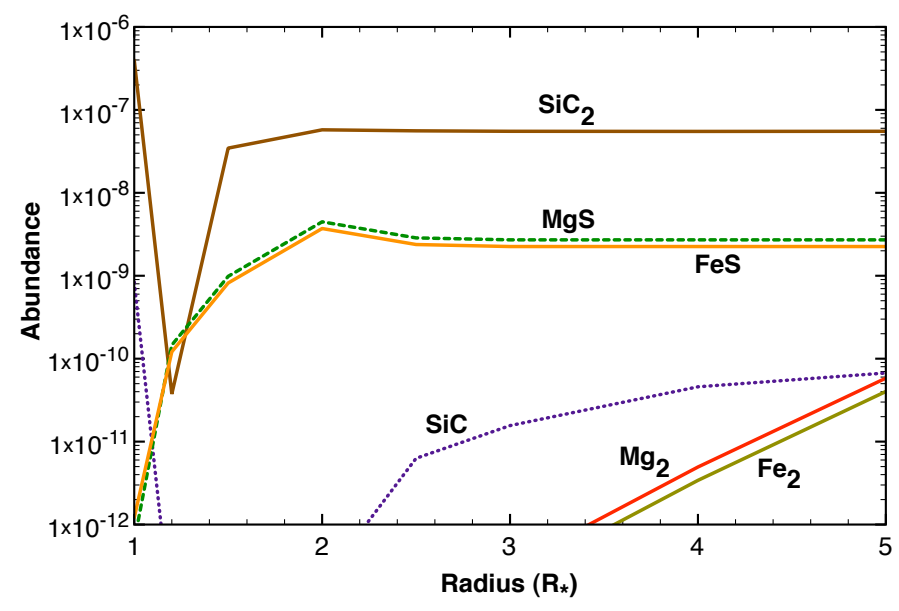

Fig. 9. Abundances (with respect to $\mathrm{H}_{2}$ ) of $\mathrm{SiC}$ and $\mathrm{SiC}_{2}$, and $\mathrm{Mg}$ - or Fe-bearing species involved in the formation of metallic clusters as a function of radius. Abundances derived by thermodynamic equilibrium at $r_{\mathrm{s}}$ are moved to radius $1 R_{\star}$ for clarity. Except for $\mathrm{SiC}_{2}$ and metal sulphide cluster precursors, abundances are quite low in the dust formation zone, and pure metal clusters do not form from the gas phase.

characterised, a few gas phase species have been identified as intermediates in the nucleation of $\mathrm{SiC}$ particles and include $\mathrm{Si}, \mathrm{C}_{2}$, and cyclic $\mathrm{SiC}_{2}$ (Fantoni et al. 1991). Silicon dicarbide, $\mathrm{SiC}_{2}$, has been detected in the inner wind of IRC +10216 by mm interferometry with abundances that range from $<5 \times 10^{-8}$ (Gensheimer et al. 1995) to $5 \times 10^{-7}$ (Lucas et al. 1995). Recent observations with HIFI onboard Herschel indicate a $\mathrm{SiC}_{2}$ abundance with respect to $\mathrm{H}_{2}$ of $2 \times 10^{-7}$ in the inner wind (Cernicharo et al. 2010a). We thus assume that the presence of $\mathrm{SiC}_{2}$ in the dust formation zone reflects the nucleation and condensation of $\mathrm{SiC}$ grains at high temperatures and densities and the role of intermediate plays by this species.

There exist no documented reaction rates for $\mathrm{SiC}_{2}$ formation and we rely on the isovalence of silicon with carbon to derive reasonable rates for specific processes. We also consider the identified nucleation routes for $\mathrm{SiC}$ clusters and the formation of $\mathrm{SiC}_{2}$ and $(\mathrm{SiC})_{2}$ clusters according to Erhart \& Albe (2005). The main production process for $\mathrm{SiC}_{2}$ in the gas phase is

$\mathrm{SiC}+\mathrm{SiC} \rightarrow \mathrm{SiC}_{2}+\mathrm{Si}$

where destruction is commanded by the reverse reaction in Eq. (10) and thermal fragmentation. We also assume that two $\mathrm{SiC}$ molecules react to form the $\mathrm{SiC}$ dimer $(\mathrm{SiC})_{2}$. The results for $\mathrm{SiC}$ and $\mathrm{SiC}_{2}$ abundances are shown in Fig. 9. The shock at $r_{\mathrm{s}}$ destroys both the $\mathrm{SiC}$ and $\mathrm{SiC}_{2}$ initially present in the photosphere under $\mathrm{TE}$, but $\mathrm{SiC}$ reforms in the post-shock gas at $1.5 R_{\star}$ and form $\mathrm{SiC}_{2}$ via the reaction in Eq. (10) and $\mathrm{SiC}$ dimers. At $5 R_{\star}$, the $\mathrm{SiC}_{2}$ abundance value agrees well with that derived by Cernicharo et al. (2010a), indicating that the molecule may be regarded as a by-product of the condensation of $\mathrm{SiC}$ clusters at small radii. The model shows that both $\mathrm{SiC}_{2}$ and $(\mathrm{SiC})_{2}$ form in large amounts as early as $1.5 R_{\star}$, and well before the aromatic formation zone $\left(2.5 R_{\star}-4 R_{\star}\right)$ discussed in Sect. 4.5. SiC clusters thus represent a high temperature condensate population independent of the synthesis of AC dust. We discuss in more detail the consequences of this situation for wind acceleration in Sect. 5 .

\subsubsection{MgS and FeS}

A strong $30 \mu \mathrm{m}$ emission band has been observed in carbonrich evolved stars at various stages of their evolution, including AGB and post-AGB stars, and planetary nebulae. Observation of the band was also reported in IRC+10216 and ascribed to solid magnesium sulphide, $\mathrm{MgS}$, for which a low radiative temperature comprised between $100 \mathrm{~K}$ and $450 \mathrm{~K}$ was derived (Goebel \& Moseley 1985; Szczerba et al. 1999; Hony et al. 2002a). A band at $23 \mu \mathrm{m}$ was observed in two carbon-rich planetary nebulae and $\mathrm{FeS}$ in the form of troilite was proposed as a possible carrier (Hony et al. 2002b). FeS is also responsible for the $23 \mu \mathrm{m}$ band detected in proto-planetary discs (Keller et al. 2002). However, there is no observational evidence that this band is present in the spectral energy distribution of C-rich evolved stars in general, including IRC+10216. Chemical models assuming TE in the inner wind predict that both $\mathrm{MgS}$ and FeS condense in carbon-rich environments (Lattimer et al. 1978; Lodders \& Fegley 1999). Keeping in mind that dust formation is not an equilibrium process in stellar outflows, we test the formation of $\mathrm{MgS}$ and $\mathrm{FeS}$ molecules as the initial gas-phase precursors of $\mathrm{MgS}$ and $\mathrm{FeS}$ grains in the inner wind. We also consider the formation of $\mathrm{Mg}$ and $\mathrm{Fe}$ - bearing molecular species and clusters. They include the pure iron and magnesium small clusters, $\mathrm{Fe}_{2}$ and $\mathrm{Mg}_{2}$ respectively, although there exists no observational evidence of pure metal clusters in AGB environments. However, pure iron grains are often proposed as a dust component of O-rich AGB winds to account for the required near-IR opacity necessary to accelerate the wind (Woitke 2006). We also consider the hydrides $\mathrm{MgH}$ and $\mathrm{FeH}$, and the gas-phase precursors to metal oxides $\mathrm{MgO}$ and $\mathrm{FeO}$.

Atomic magnesium is an alkaline earth metal that primarily reacts with oxygen compounds (e.g., $\mathrm{H}_{2} \mathrm{O}, \mathrm{O}_{2}, \mathrm{NO}, \mathrm{O}_{3}$ ) to form magnesium oxides. Although reactions with sulphurbearing compounds are not documented, we expect similar types of reaction between $\mathrm{Mg}$ and $\mathrm{SO}$ to those between $\mathrm{Mg}$ and $\mathrm{O}_{2}$, owing to the isovalence of sulphur with oxygen. We therefore assume that $\mathrm{Mg}$ will react with $\mathrm{SO}$ according to the reaction

$\mathrm{Mg}+\mathrm{SO} \rightarrow \mathrm{MgS}+\mathrm{O}$

Using again the isovalence of sulphur with oxygen, we assume that a reaction similar to the reaction in Eq. (11) triggers the formation of molecular FeS. For the formation of iron monoxide, $\mathrm{FeO}$, and magnesium monoxide, $\mathrm{MgO}$, reactions of atomic $\mathrm{Fe}$ and $\mathrm{Mg}$ with $\mathrm{O}_{2}$ are considered. These processes have been extensively studied (e.g., Akhmadov et al. 1988) and their rates well-documented. For both sulphides, we also consider the following radiative association reaction as a possible production channel

$\mathrm{X}+\mathrm{S} \rightarrow \mathrm{XS}+\mathrm{h} v$

where $X=\mathrm{Mg}$ or Fe. Studies by Kimura et al. $(2005 \mathrm{a}, \mathrm{b})$ that explore various formation routes to $\mathrm{MgS}$ and $\mathrm{FeS}$ in the laboratory show very efficient synthesis from the reaction of the two $\mathrm{Mg}(\mathrm{Fe})$ and $\mathrm{S}$ gaseous phases in gas flash evaporation methods, and support the occurrence of the reaction in Eq. (12) and its termolecular analogue process. The formation of $\mathrm{Fe}_{2}$ is described by the reaction

$\mathrm{Fe}+\mathrm{Fe}+\mathrm{M} \rightarrow \mathrm{Fe}_{2}+\mathrm{M}$,

where $M$ is the gas collider. A rate for the reaction in Eq. (13) was derived by Giesen et al. (2003) in their study of pure iron cluster formation at high temperatures. The radiative association 
reaction between two $\mathrm{Fe}$ atoms is also included. Similar types of processes and rates are considered for the synthesis of $\mathrm{Mg}_{2}$. We assume that the reverse reactions of all chemical pathways are the only destruction processes operating on $\mathrm{Fe}_{2}$ and $\mathrm{Mg}_{2}$.

Abundances with respect to $\mathrm{H}_{2}$ for these species are shown in Fig. 9, except for $\mathrm{FeO}$ and $\mathrm{MgO}$ which have negligible abundances in the inner wind $\left(x(\mathrm{MgO}, \mathrm{FeO}) \sim 1 \times 10^{-18}\right)$. Apart from $\mathrm{MgS}$ and $\mathrm{FeS}, \mathrm{Mg}$ - and Fe-bearing species have very low abundances. Specifically, $\mathrm{Fe}$ and $\mathrm{Mg}$ do not form pure metal clusters as $\mathrm{Fe}_{2}$ and $\mathrm{Mg}_{2}$ have low abundances in the dust formation zone. According to our model, most of the $\mathrm{Mg}$ and $\mathrm{Fe}$ initially present in the photosphere and at $r_{\mathrm{s}}$ stays in atomic form. A moderate amount of $\mathrm{Mg}$ and $\mathrm{Fe}$ is first included in the hydrides $\mathrm{MgH}$ and $\mathrm{FeH}$ (see Fig. 3) but are preferentially included into $\mathrm{MgS}$ and FeS at $r \geq 2 R_{\star}$ from the reaction of $\mathrm{Mg}$ and Fe with SO following the reaction $n$ Eq. (11). Similar to $\mathrm{H}_{2} \mathrm{O}$, sulphur monoxide formation is induced by the release of oxygen atoms in the collisional dissociation of $\mathrm{CO}$ in the hot post-shock gas.

An upper limit to the total mass of solid $\mathrm{MgS}$ and FeS produced is derived assuming that all gas-phase $\mathrm{MgS}$ and $\mathrm{FeS}$ are depleted into clusters and grains once the gas has reached the temperature regime $(T<450 \mathrm{~K})$ derived by Goebel \& Moseley (1985). The mass limit for both $\mathrm{MgS}$ and $\mathrm{FeS}$ is at most $\sim 0.2 \%$ of the carbon dust mass formed between $r_{\mathrm{s}}$ and $5 R_{\star}$. When modelling the spectral energy distribution of IRC +10216 , Ivezić \& Elitzur (1996) derive a dust composition where $\mathrm{MgS}$ accounts for less than $10 \%$ by mass. For the carbon-rich post-AGB star HD 56126, Hony et al. (2003) found that a MgS mass of $\sim 2 \%$ of the carbon dust mass is necessary to account for the $30 \mu \mathrm{m}$ band flux. One would thus expect the MgS mass to represent at most a few percent of the AC dust mass in the inner wind of carbon stars. This required mass is higher than our upper limit by a factor of ten. This discrepancy may be the result of several uncertainties in the sulphur chemistry or may point to either a nucleation process for $\mathrm{MgS}$ that does not occur in the gas phase or to a different carrier for the $30 \mu \mathrm{m}$ band. These various aspects are discussed in Sect. 5.

Gas-phase FeS follows an abundance trend similar to $\mathrm{MgS}$, as seen in Fig. 9, because we assumed that the FeS and $\mathrm{MgS}$ chemistries were alike. Both $\mathrm{Fe}$ and $\mathrm{Mg}$ are mainly in atomic form and have very similar abundances at TE in the photosphere. Both solid $\mathrm{MgS}$ and $\mathrm{FeS}$ possess identical clustering structures going from $(\mathrm{XS})_{2}(\mathrm{X}=\mathrm{Mg}, \mathrm{Fe})$ with its planar rhombic structure to $(\mathrm{XS})_{4}$, which quickly reaches a distorted cubic structure. However, MgS clusters are unstable in O-rich environments, contrarily to FeS clusters. It was proposed by Begemann et al. (1994) that composite solid sulphides including both $\mathrm{Mg}$ and $\mathrm{Fe}$ could satisfactorily reproduce the $30 \mu \mathrm{m}$ band in IRC +10216 . In particular, a magnesium-iron sulphides whose composition ranged from $\mathrm{Mg}_{0.9} \mathrm{Fe}_{0.1} \mathrm{~S}$ to $\mathrm{Mg}_{0.5} \mathrm{Fe}_{0.5} \mathrm{~S}$ provided the closest matches to the band. Such a carrier could indeed be synthesized in the dust formation zone of IRC+10216 in view of the Feand $\mathrm{Mg}$-bearing species that form, and the presence of both gasphase $\mathrm{MgS}$ and FeS.

As pointed out before, most of the $\mathrm{Mg}$ and $\mathrm{Fe}$ in the inner wind region is in atomic form. High resolution observations of optical absorption lines of several metals by Mauron \& Huggins (2010) for IRC+10216 also appear to detect atomic metals in the gas phase, in direct contradiction with TE condensation models for which all metals are depleted in a solid phase once the condensation temperature of the solid is reached in the wind. However, the derived abundances for iron and calcium atoms highlighted some degree of depletion relative to the solar abundance values. According to this study, the depletion cannot result

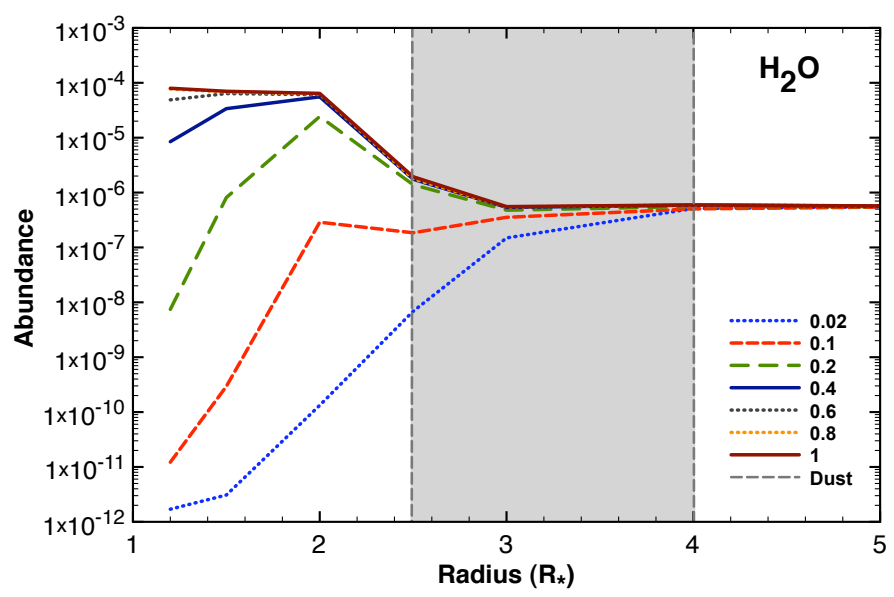

Fig. 10. $\mathrm{H}_{2} \mathrm{O}$ abundances (with respect to total gas) as a function of radius and pulsation phase (legend). Phases 0.8 and 1 are indistinguishable. The grey shaded area corresponds to the zone where benzene forms and aromatics grow to form carbon dust (see Sect. 4.5).

from the trapping in a molecular phase, as corroborated by the present results where the abundances of metal-bearing species are always less than $1 \times 10^{-6}$. The partial depletion of iron and calcium must thus result from either the incorporation of freeflying $\mathrm{Fe}$ and $\mathrm{Ca}$ atoms in the inner wind during the $\mathrm{AC}$ dust condensation process at $r \leq 5$, or the adsorption of these atoms on the surface of dust grains at the lower gas temperatures encountered at larger envelope radii.

\subsection{Line variability with time}

Our model predicts that specific molecular abundances have a strong time-dependence. Some molecular species indeed show a strong variability in their abundances as a function of time or phase of the pulsation period. The abundance of water is a good example of such a variability and its abundance with respect to $\mathrm{H}_{2}$ as a function of radius and pulsation phase is shown in Fig. 10. At $2 R_{\star}, \mathrm{H}_{2} \mathrm{O}$ abundances span almost six orders of magnitude over one pulsation period ( $P=650$ days $)$, and this variation should be reflected in the intensity of its high-energy transitions. In these deep layers, the transitions are mainly pumped by IR radiation. Apart from the intrinsic variability of the stellar flux with pulsation phase that affects all molecules, the large variations in abundances should have some impact on the high excitation-line fluxes. These changes in abundances are the consequence of the post-shock non-equilibrium chemistry and the destruction of molecular species in the hot post-shock gas at early phases.

Destruction is more or less severe depending on the species and not all molecules behave like $\mathrm{H}_{2} \mathrm{O}$. For example, with its strong molecular bond, $\mathrm{CO}$ does not experience such variations and shows a rather constant abundance distribution with radius and pulsation phase. Some species (e.g., SiO), show a time variation in abundance that increases in amplitude from large radii to small radii, but where the variation amplitudes do not span more than one order of magnitude. Finally, other molecules (e.g., $\mathrm{SiC}_{2}$ ) show average amplitude variations (of three orders of magnitude) at small radii but reach time-independent abundances deep inside the inner wind $\left(\sim 2.4 R_{\star}\right)$. Although the impact of time-varying abundances on the line fluxes is difficult to quantify without a proper radiative transfer model, we would expect some species to show little line variability (e.g. CO) but others to be prone to moderate variability (e.g., $\mathrm{SiO}$ ) and undergo 
large line-flux changes with time (e.g., $\mathrm{H}_{2} \mathrm{O}$ ). Clearly water is an excellent tracer of time variability of high-energy molecular transitions and as such, an excellent indicator of shock activity and shock-induced chemistry in the dust formation zone.

\section{Summary and discussion}

We have modelled the inner wind of the carbon star IRC+10216 assuming the periodic propagation of pulsation-driven shocks between $1 R_{\star}$ and $5 R_{\star}$ and considering a complete gas-phase chemistry that encompasses several chemical families. These shocks trigger a non-equilibrium chemistry in the hot post-shock gas that leads to the formation of molecules and dust precursors. The study points to the following new results and trends applicable in general to carbon stars:

- The model confirms the presence of a group of molecules, namely $\mathrm{CO}, \mathrm{HCN}, \mathrm{SiO}, \mathrm{CS}$, and $\mathrm{H}_{2} \mathrm{O}$, that efficiently form between $1 R_{\star}$ and $5 R_{\star}$. These species are expected to be present, albeit in different quantities, in the inner wind of all AGB stars, regardless of their $\mathrm{C} / \mathrm{O}$ ratio, as already proposed by Cherchneff (2006, 2011a). The derived abundance values agree well with available observations. In particular, the dissociation of $\mathrm{CO}$ by collisions in the immediate post-shock gas triggers the formation of atomic $\mathrm{O}, \mathrm{OH}, \mathrm{SiO}$, and $\mathrm{H}_{2} \mathrm{O}$.

- We have found that some hydrides form a new category of abundant and stable species in the inner wind. These include $\mathrm{AlH}, \mathrm{HCl}$, and $\mathrm{HF}$. Other hydrides do form in large amounts at $r_{\mathrm{s}}$ but are rapidly converted in the dust formation zone into chlorine-bearing species, leaving the wind acceleration region with low abundances. The most abundant hydrides will be released to the intermediate envelope and participate in an active chemistry at larger radii.

- Once formed from the reaction of $\mathrm{H}_{2}$ with $\mathrm{Cl}, \mathrm{HCl}$ is the production agent of other Cl-bearing species such as $\mathrm{AlCl}$, $\mathrm{NaCl}$, or $\mathrm{KCl}$. The formation chemistry of chlorine-bearing species is thus independent of the $\mathrm{C} / \mathrm{O}$ ratio of the stellar photosphere, and Cl-bearing molecules including $\mathrm{HCl}$, $\mathrm{NaCl}$, and $\mathrm{KCl}$ should also be present in O-rich $\mathrm{AGB}$ and supergiant stars, albeit with different abundances. $\mathrm{AlCl}$ is expected to have a lower abundance in the dust formation zone of O-rich sources because of the Al depletion in gas- or solidphase metal oxide species.

- There exists a specific zone that extends from $2.5 R_{\star}$ to $4 R_{\star}$, where the closure of $\mathrm{C}_{6} \mathrm{H}_{6}$ ring occurs through the recombination of two propargyl radicals, with a $\mathrm{C}_{6} \mathrm{H}_{6}$ abundance peaking at $3 R_{\star}$. The available $\mathrm{C}_{2} \mathrm{H}_{2}$ abundances in the gas phase are high enough to secure growth to PAHs such as $\mathrm{C}_{24} \mathrm{H}_{12}$. These large PAHs will consecutively coalesce and coagulate to form $\mathrm{AC}$ dust. The estimated total dust-to-gas mass ratio spans the value range $1.2-5.8 \times 10^{-3}$ and closely agrees with existing values derived from observations of IRC +10216 . Within this zone, $\mathrm{SiC}_{2}$ molecules efficiently form as by-products of the synthesis of $\mathrm{SiC}$ clusters. Some $\mathrm{MgS}$ and $\mathrm{FeS}$ species are also produced in the gas phase but their abundances are too low to account for the 26-30 $\mu \mathrm{m}$ emission band.

- The shock-induced scenario predicts a time-variability for some molecular abundances over a pulsation period (e.g. $\mathrm{H}_{2} \mathrm{O}$ and $\mathrm{SiO}$ ) that should induce a time variability in their high excitation line fluxes. Other species show either negligible changes in abundances (e.g., CO), or small changes that do not affect the molecular line intensity (e.g., $\mathrm{SiC}_{2}$ ). This predicted time variability is the direct result of the destruction of species in the hot shocked gas layers. Observations of the high-energy transitions of these specific species at different epochs of the pulsation period would help us to confirm the predicted time variability, and assess the impact of shocks on the gas chemistry.

As reported in Sect. 4.5, we have found that the formation of $\mathrm{PAH}$ molecules, and both their coalescence and growth to AC dust take place in a specific radius range. The growth of benzene, $\mathrm{C}_{6} \mathrm{H}_{6}$, to coronene, $\mathrm{C}_{24} \mathrm{H}_{12}$, via the HACA mechanism is expected to consume a large part of the benzene rings synthesised at these radii. At radii larger than $4 R_{\star}$, inspection of Table 4 shows that some benzene rings still form and can grow to larger aromatic species as the $\mathrm{C}_{2} \mathrm{H}_{2}$ reservoir is still large. However, the lower gas densities and temperatures should hinder the coalescence of PAHs to form large graphene structures. A population of free-flying PAHs not incorporated in AC dust should thus be expelled to larger radii once the wind is fully acccelerated. The so-called unidentified infrared bands are observed in carbon stars that are part of binary systems (Speck \& Barlow 1997; Boersma et al. 2006). For the carbon star TU Tau, the UV radiation field of the blue companion could excite the aromatics present in the carbon star wind. These excited PAHs might include the free aromatics that are synthesised beyond the aromatic formation zone highlighted in this study.

We have also found that $\mathrm{SiC}$ dimers form at $\sim 1.5 R_{\star}$, i.e., far smaller radii than the aromatic growth region, which implies that there is an independent population of $\mathrm{SiC}$ clusters at small radii. Owing to the extinction properties of $\mathrm{SiC}$ dust and a significant decrease in the Planck mean of its extinction efficiency for temperatures corresponding to the effective temperatures of AGB stars, the dust experiences an inverse greenhouse effect for a radiation field characteristic of carbon stars (Gilman 1974; McCabe 1982; Yasuda \& Kozasa 2012). Since the pressure force acting on dust grains is directly proportional to the Planck mean of the extinction efficiency, most of the acceleration of the wind is provided by AC dust grains (Cherchneff et al. 1991). On the other hand, the inner $\mathrm{SiC}$ cluster population should experience a minor radiation pressure force and lags behind the AC clusters when expelled to larger radii. This situation may be reflected in the results of studies of meteorites. Pre-solar SiC grains bearing the isotopic fingerprint of the AGB s-process are not included in graphite spherules that have an AGB origin but form a separate pre-solar grain population (Hynes et al. 2007). This isolation of the $\mathrm{SiC}$ presolar grains may directly result from the non-equilibrium chemistry in the post-shock gas that produces two main dust populations, namely $\mathrm{SiC}$ and $\mathrm{AC}$ grains, at two distinct positions in the wind acceleration zone.

No firm conclusions about the production of $\mathrm{MgS}$ or $\mathrm{Mg}-\mathrm{FeS}$ dust grains in IRC+10216 can be drawn from the present results, as they can be interpreted in many ways. Firstly, the chemical model may underestimate the $\mathrm{MgS}$ and $\mathrm{FeS}$ abundances by a factor of ten or more because too much atomic sulphur is trapped in $\mathrm{SiS}$, as indicated by the slightly higher SiS abundances listed in Table 3 relative to those derived from observations. A small amount of S not locked in SiS would result in higher SO abundances and in a larger amount of $\mathrm{MgS}$ and $\mathrm{FeS}$ owing to the large reservoirs of free atomic $\mathrm{Mg}$ and $\mathrm{Fe}$ in the inner wind. The $\mathrm{MgS}$ and FeS clusters would then be produced from the gas phase at $r \geq 2 R_{\star}$, and the resulting estimated $\mathrm{MgS}$ dust mass could reach the few percent of AC dust mass necessary to explain the $30 \mu \mathrm{m}$ band. That the present model forms gas-phase $\mathrm{FeS}$ and $\mathrm{MgS}$ with similar efficiencies indicate that it may form composite $\mathrm{Mg}$-Fe sulphide clusters instead of pure $\mathrm{MgS}$ clusters, 
Table A.1. The chemical reaction network ordered by type of reactions.

\begin{tabular}{llllcccc}
\hline \hline & & & Termolecular & & & \\
3B1 & $\mathrm{H}+\mathrm{H}+\mathrm{H}_{2}$ & $\longrightarrow$ & $\mathrm{H}_{2}+\mathrm{H}_{2}$ & $8.85 \times 10^{-33}$ & -0.6 & 0 & NIST \\
3B2 & $\mathrm{H}+\mathrm{H}+\mathrm{H}$ & $\longrightarrow$ & $\mathrm{H}_{2}+\mathrm{H}$ & $8.82 \times 10^{-33}$ & 0 & 0 & NIST \\
3B3 & $\mathrm{H}+\mathrm{H}+\mathrm{He}$ & $\longrightarrow$ & $\mathrm{H}_{2}+\mathrm{He}$ & $4.96 \times 10^{-33}$ & 0 & 0 & NIST \\
3B4 & $\mathrm{H}+\mathrm{O}+\mathrm{M}$ & $\longrightarrow$ & $\mathrm{OH}+\mathrm{M}$ & $4.36 \times 10^{-32}$ & -1.0 & 0 & NIST \\
\hline
\end{tabular}

Notes. The full table is available at the CDS. The reaction rate is given in the Arrhenius form $A \times(T / 300)^{n} \times \exp \left(-E_{a} / T\right)$, where $T$ is the temperature in Kelvin. The related parameters are given in each column as follows: Col. 1 - reaction number; Col. 2 - reactants and products; Col. 3 - A parameter (in $\mathrm{cm}^{3} \mathrm{~s}^{-1}, \mathrm{~cm}^{6} \mathrm{~s}^{-1}$, and $\mathrm{s}^{-1}$ for bimolecular, trimolecular, and unimolecular processes, respectively); Col. $4-n$ parameter; Col. 5 - activation barrier $E_{a}$ in Kelvin: Col. 6 - rate reference.

as proposed by Begemann et al. (1994). According to Kimura et al. (2005a,b), a MgS (FeS) nucleation from the gas phase produces spherical cubic $\mathrm{MgS}(\mathrm{FeS})$ clusters instead of the elongated network-like grains that form when gas-surface reactions are involved in the nucleation process. Hony et al. (2002b) studied the effect of dust shape and temperature on the band shift in wavelength with a shift towards $26 \mu \mathrm{m}$ when spherical and hot grains were considered. The emission band in IRC +10216 clearly peaks around $27 \mu \mathrm{m}$ in the ISO spectrum, pointing to a possible formation pathway from gas-phase chemistry, as we have described in the present study. Secondly, if the MgS (FeS) abundances are indeed low in the dust formation zone, they point to: 1) a synthesis mechanism for $\mathrm{MgS}$ or $\mathrm{Mg}-\mathrm{FeS}$ involving gassurface processes, or 2) alternative carriers for the $30 \mu \mathrm{m}$ band. However, if $\mathrm{MgS}$ grain formation occurs at lower temperatures and on the surface of already produced dust grains (see the comprehensive studies by Men'shchikov et al. 2001 and Zukhovska \& Gail 2008), their growth is hindered by the lack of available atomic sulphur, which is chiefly depleted in SiS and CS in the dust production zone. Therefore, a formation scenario involving surface chemistry would also require a mechanism that could return sulphur to the gas phase just after the acceleration of the outflow. Another explanation is that $\mathrm{MgS}$ is not the carrier of the $30 \mu \mathrm{m}$ band. A critical assessment of all previous MgS studies was made by Zhang et al. (2009) who pointed out that the mass of $\mathrm{MgS}$ derived from the emission at $30 \mu \mathrm{m}$ violated the available abundances of $\mathrm{Mg}$ and $\mathrm{S}$ in the stellar atmospheres, owing to the use of improper optical constants for $\mathrm{MgS}$ in the optical and UV wavelength domains. Alternative solids have been proposed (e.g., hydrogenated amorphous carbon, HAC, Grishko et al. 2001). A fresh reinvestigation of the carrier of the band in IRC +10216 would be extremely useful coupled to observations of high energy transitions of sulphur-bearing species to constrain the sulphur reservoir in the dust formation zone.

Finally, the results presented in this study are not unique to IRC +10216 and similar trends should apply to other carbon stars as well. For the specific case of water, $\mathrm{H}_{2} \mathrm{O}$ has now been detected in several carbon stars (Neufeld et al. 2011b) where formation processes similar to those described in Cherchneff (2011a) and in the present study take place. However, the $\mathrm{H}_{2} \mathrm{O}$ abundance certainly varies from source to source depending on the various parameters that are entangled with its formation in a complex way. For example, the shock strength affects both the destruction of molecules and the creation of free atomic oxygen. Therefore, one would expect lower water abundances to be created by a mild shock than a strong shock, but the opposite actually occurs. We have modelled the chemistry induced by a $10 \mathrm{~km} \mathrm{~s}^{-1}$ shock at $r_{\mathrm{s}}$ and compared our results with those of the $20 \mathrm{~km} \mathrm{~s}^{-1}$ shock, finding that more $\mathrm{SiO}$ and $\mathrm{H}_{2} \mathrm{O}$ molecules were produced. Because less $\mathrm{CO}$ is destroyed by a mild shock, the $\mathrm{SiO}$ formation depends on the reaction of $\mathrm{Si}$ with $\mathrm{CO}$. Therefore, the $\mathrm{OH}$ radical is free to form $\mathrm{H}_{2} \mathrm{O}$, and combined with the less efficient destruction of molecules in the post-shock gas, more water is formed. Hence, the many parameters affecting the formation of water in carbon stars include the shock strength as well as the chemical composition, both the photospheric gas density and temperature, the gas-phase chemistry of the Si and $\mathrm{S}$ chemical families, and the amount and type of dust that forms. Water abundances is thus expected to vary greatly in carbon stars despite its synthesis in these objects having been proven both observationally and theoretically. A similar conclusion may be drawn for other species such as $\mathrm{SiO}$. Combined observations of several high excitation transitions of $\mathrm{H}_{2} \mathrm{O}$ and $\mathrm{SiO}$ molecules would be very instructive in this regard to more clearly understand the chemical processes responsible for the formation of O-bearing species and characterise the water content of carbon stars on a global scale.

Acknowledgements. The author thanks the two anonymous referees for their useful comments that helped to improve the manuscript, A. Tielens for constructive remarks, and D. Gobrecht for providing the TE calculation estimates.

\section{References}

Agúndez, M. 2009, Ph.D. Thesis 98

Agúndez, M., \& Cernicharo, J. 2006, ApJ, 650, 374

Agúndez, M., Cernicharo, J., \& Guélin, M. 2007, A\&A, 662, L91

Agúndez, M., Cernicharo, J., \& Guélin, M. 2010, ApJ, 724, L133

Agúndez, M. Cernicharo, J., Waters, L. B. F. M., et al. 2011, A\&A, 533, L6

Akhmadov, U. S., Zaslomko, I. S., \& Smirnov, V. N. 1988, Kin. Cata., 29, 251

Asplund, M., Grevesse, N., Sauval, A. J., \& scott, P. 2009, ARA\&A, 47, 481

Begemann, B., Dorschner, T., Henning, T., et al. 1994, ApJ, 423, L71

Bertschinger, E., \& Chevalier, R. A. 1985, ApJ, 299, 167

Boersma, C., Hony, S., \& Tielens, A. G. G. M. 2006, A\&A, 447, 213

Bowen, G. H. 1988, ApJ, 329, 299

Bulgakov, A. V., Bobrenok, O. F., Kosyakov, V. I., et al. 2002, Phys. Solid State, 44, 617

Cernicharo, J., Waters, L. B. F. M., Decin, L., et al. 2010a, A\&A, 521, L10

Cernicharo, J., Decin L., Barlow, M. J., et al. 2010b, A\&A, 518, L136

Cernicharo, J., Agúndez, M.. Kahane, C., et al. 2011, A\&A, 529, L3

Cherchneff, I. 1996, in Molecules in astrophysics: probes \& processes, ed. E. van Dishoeck, Proc. IAU Symp., 178, 469

Cherchneff, I. 2006, A\&A, 456, 1001

Cherchneff, I. 2011a, A\&A, 256, L11

Cherchneff, I. 2011b, EAS, 46, 177

Cherchneff, I., Barker, J. R., \& Tielens, A. G. G. M. 1991, ApJ, 377, 541 Cherchneff, I., Barker, J. R., \& Tielens, A. G. G. M. 1992, ApJ, 401, 269 Cohen, N., \& Westberg, K. R. 1983, J. Phys. Chem. Ref. Data, 12, 531

Decin, L., Cherchneff, I., Hony, S., et al. 2008, A\&A, 480, 431

Decin, 1., Cernicharo, J., Barlow, M. J., et al. 2010a, A\&A, 518, L143

Decin, L., Agúndez, M., Barlow, M. J., et al. 2010b, Nature, 467, 64

Deguchi, S., \& Goldsmith, P. F. 1985, Nature 317, 336

Duari, D., Cherchneff, I., \& Willacy, K. 1999, A\&A, 341, L47

Erhart, P., \& Albe, K. 2005, Adv. Engin. Mat., 7, 937

Fantoni, R., Bijnen, F., Djuric, N., \& Piccirillo, S. 1991, Appl. Phys. B., 52, 176 Fonfría, J. P., Cernicharo, J., Richter, M. J., \& Lacy, J. 2008, ApJ, 673, 445

Ford, K. S. E., Neufeld, D. A., Schilke, P., \& Melnick, G. J. 2004, ApJ, 614, 990 Fox, M. W., \& Wood, P. R. 1985, ApJ, 297, 455 
Frenklach, M., Clary, D. W., Gardiner, W. C., Jr., \& Stein, S. E. 1984, 20th Symp. (Int.) on Combustion, The Combustion Institute, 887

Gensheimer, P. D., Likkel, L., \& Snyder, L. E. 1995, ApJ, 439, 445

Giesen, A., Herzler, J., \& Roth, P. 2003, J. Phys. Chem., 107, 5202

Gilman, R. C. 1974, ApJ, 268, 397

Goebel, J. H., \& Moseley, S. H. 1985, ApJ, 290, L35

Grishko, V. I., Tereszchuk, K., Duley, W. W., \& Bernath, P. 2001, ApJ, 558, L129 Groenewegen, M. 1998, A\&A, 338, 491

Guélin, M., Cernicharo, J., Paubert, G., \& Turner, B. E. 1990, A\&A, 230, L9

Halfen, D. T., Clouthier, D. J., \& Ziurys, L. M. 2008, ApJ, 677, L101

He, J. H., Dinh-V-Trung, Kwok, S., et al. 2008, ApJS, 177, 275

Hony, S., \& Bouwman, J. 2004, A\&A, 413, 981

Hony, S., Waters, L. B. F. M., \& Tielens, A. G. G. M. 2002a, A\&A, 390, 533

Hony, S., Bouwman, J., Keller, L. P., \& Waters, L. B. F. M. 2002b, A\&A, 393, L103

Hony, S., Tielens, A. G. G. M., Waters, L. B. F. M., \& de Koter, A. 2003, A\&A, 402, 211

Husain, D., \& Marshall, P. 1986, Int. J. Chem. Kin., 18, 83

Hynes, K. M., Croat, T. K., \& Bernatowicz, T. J. 2007, LPI, 38, 1693

Ivezić, Z., \& Elitzur, M. 1996, MNRAS, 279, 1011

Justtanont, K., de Jong, T., Helmich, F. P., et al. 1996, A\&A, 315, L217

Keady, J. J., Hall, Donald N. B., \& Ridgway, S. T. 1988, ApJ, 326, 832

Keller, L. P., Hony, S. Bradley, J. P., et al. 2002, Nature, 417, 158

Kimura, Y., Kurumada, M., Tamura, K., et al. 2005a, A\&A, 442, 507

Kimura, Y., Tamura, K., Koike, C., et al. 2005b, Icarus, 177, 280

Krestinin, A. V. 2000, Combustion \& Flame, 121, 513

Lattimer, J. M., Schramm, D. N., \& Grossman, L. 1978, ApJ, 219, 230

Lodders, K., \& Fegley, B. 1999, in Asymptotic Giant Branch Stars, IAU Symp., 191, 279

Lucas, R., Guélin, M., Kahane, C., et al. 1995, Ap\&SS, 224, 293

Mauron, N., \& Huggins, P. 2010, A\&A, 513, A31

McCabe, E. M. 1982, MNRAS, 200, 71

McCabe, E. M., Smith, R. C., \& Clegg, R. E. S. 1979, Nature, 281, 263

Melnik, G. J., Neufeld, D. A., Ford, K. E. S., et al. 2001, Nature, 412, 160

Men'shchikov, A. B., Balega, Y., Blöcker, T., et al. 2001, A\&A, 368, 497

Milam, S. N., Apponi, A. J., Woolf, N. J., \& Ziurys, L. M. 2007, ApJ, 668, L131

Milam, S. N., Halfen, D. T., Tenenbaum, E. D., et al. 2008, ApJ, 684, 618
Nejad, L. A. M., \& Millar, T. J. 1988, MNRAS, 230, 79

Neufeld, D, González-Alfonso, E. Melnick, G., et al. 2010, A\&A, 521, L5

Neufeld, D., González-Alfonso, E. Melnick, G., et al. 2011a, ApJ, 727, L28

Neufeld, D., González-Alfonso, E. Melnick, G., et al. 2011b, ApJ, 727, L29

Olofsson, H. 2008, Phys. Scri., 133, 014028

Olofsson, H., Johansson, L. E. B., Hjamarson, Å., \& Nguyen-Quang-Rieu 1982, A\&A, 107, 128

Ozin, G. A., \& McCaffrey, J. G. 1984, J. Phys. Chem, 88, 645

Patel, N., Young, K. H., Brünken, S., et al. 2009, ApJ, 692, 1205

Perrin, G., Ridgway, S. T., Mennesson, B., et al. 2004, A\&A, 426, 279

Ridgway, S. T., \& Keady, J. J. 1981, Phil. Trans. R. Soc. Lond., A, 303, 497

Ridgway, S. T., \& Keady, J. J. 1988, ApJ, 326, 843

Schöier, F. L., Olofsson, H., \& Lundgren, A. A. 2006, A\&A, 454, 247

Schöier, F. L., Bast, J., Olofsson, H., \& Lindqvist, M. 2007, A\&A, 473, 871

Shinnaga, H., Young, K. H., Tilanus, R. P. J., et al. 2009, ApJ, 698, 1924

Speck, A., \& Barlow, M. J. 1997, Ap\&SS, 251, 115

Speck, A., Barlow, M. J., \& Skinner, C. J. 1997, MNRAS, 288, 431

Szczerba, R., Henning, T., Volk, K., et al. 1999, A\&A, 345, L39

Tenenbaum, E. D., \& Ziurys, L. M. 2010, ApJ, 712, L93

Tenenbaum, E. D., Dodd, J. L., Milam, S. N., Woolf, N. J., \& Ziurys, L. M. 2010, ApJS, 190, 348

Treffers, R., \& Cohen, M. 1974, ApJ, 188, 545

Tsuji, T. 1973, A\&A, 23, 411

Tsuji, T., Ohnaka, K., Aoki, W., \& Yamamura, I. 1997, A\&A, 320, L1

Willacy, K. 2004, ApJ, 600, L87

Willacy, K., \& Cherchneff, I. 1998, A\&A, 330, 676

Winters, J. M., Dominik, C., \& Sedlmayr, E. 1994, A\&A, 288, 255

Woitke, P. 2006, A\&A, 460, L9

Yasuda, Y., \& Kozasa, T. 2012, ApJ, 745, 159

Zhang, K., Jiang, B. W., \& Li, A. 2009, ApJ, 702, 680

Zhukovska, S., \& Gail, H.-P. 2008, A\&A, 486, 229

Zinner, E. 2007, In Treatise on Geochemistry, eds. H. D. Holland \& K. K. Turekian (Oxford: Elsevier Ltd.), 1.02, 1

Ziurys, L. M., Milam, S. N., Apponi, A. J., \& Woolf, N. J. 2007, Nature, 447, 1094

Ziurys, L. M., Tenenbaum, E. D., Pulliam, R. L., Woolf, N. J., \& Milam, S. N. 2009, ApJ, 695, 1604 